\title{
Quasi-Maximum Likelihood and The Kernel Block Bootstrap for Nonlinear DYNAMIC MODELS*
}

\author{
Paulo M.D.C. Parente \\ REM and CEMAPRE \\ ISEG, School of Economics and Management, Universidade de Lisboa \\ pparente@iseg.ulisboa.pt \\ RichaRd J. SMITH \\ cemmap, U.C.L and I.F.S. \\ Faculty of Economics, University of Cambridge \\ Department of Economics, University of Melbourne \\ ONS Economic Statistics Centre of Excellence \\ rjs27@econ.cam.ac.uk
}

This Draft: October 2020

\begin{abstract}
This paper applies a novel bootstrap method, the kernel block bootstrap, to quasi-maximum likelihood estimation of dynamic models with stationary strong mixing data. The method first kernel weights the components comprising the quasi-log likelihood function in an appropriate way and then samples the resultant transformed components using the standard " $m$ out of $n$ " bootstrap. We investigate the first order asymptotic properties of the kernel block bootstrap method for quasi-maximum likelihood demonstrating, in particular, its consistency and the first-order asymptotic validity of the bootstrap approximation to the distribution of the quasi-maximum likelihood estimator. A set of simulation experiments for
\end{abstract}

${ }^{*}$ We would like to thank a Co-Editor and the referees for their helpful and constructive comments on the previous version of the paper. The authors are also grateful to participants for their comments on earlier versions of this paper presented at the 2020 ANZESG Meeting, London School of Economics, Monash University, Universidad de Alicante and the Universities of Cambridge, Melbourne, Queensland, Sydney and York. Paulo Parente is grateful for the financial support of Fundação para a Ciência e a Tecnologia (Project PTDC/IIM-ECO/3097/2014).

Address for correspondence: Paulo M.D.C. Parente, ISEG, School of Economics and Management, Universidade de Lisboa, Rua do Quelhas 6, 1200-781 Lisboa, Portugal.

This is the author manuscript accepted for publication and has undergone full peer review but has not been through the copyediting, typesetting, pagination and proofreading process, which may lead to differences between this version and the Version of Record. Please cite this article as doi: $10.1111 /$ jtsa.12573

This article is protected by copyright. All rights reserved. 
the mean regression model illustrates the efficacy of the kernel block bootstrap for quasi-maximum likelihood estimation.

JEL Classification: C14, C15, C22

Keywords: Bootstrap; heteroskedastic and autocorrelation consistent inference; quasi-maximum likelihood estimation.

\section{INTRODUCTION}

This paper applies the kernel block bootstrap (KBB), proposed in Parente and Smith (2018), PS henceforth, to provide accurate confidence regions for a sub-vector or a smooth function of the parameter vector in the quasi-maximum likelihood (QML) framework with stationary and weakly dependent data. The basic idea underpinning KBB arises from earlier papers, see, e.g., Kitamura and Stutzer (1997) and Smith (1997, 2011), which recognise that a suitable kernel function-based weighted transformation of the observational sample with weakly dependent data preserves the large sample efficiency for randomly sampled data of (generalised) empirical likelihood, (G)EL, methods. In particular, the mean of and, moreover, the standard random sample variance formula applied to the transformed sample are respectively consistent for the population mean [Smith (2011, Lemma A.1, p.1217)] and a heteroskedastic and autocorrelation (HAC) consistent and automatically positive semidefinite estimator for the variance of the standardized mean of the original sample [Smith (2005, Section 2, pp.161-165, and 2011, Lemma A.3, p.1219)].

In a similar spirit, KBB applies the standard " $m$ out of $n$ " nonparametric bootstrap, originally proposed in Bickel and Freedman (1981), to the transformed kernel-weighted data. PS demonstrate, under appropriate conditions, the large sample validity of the KBB estimator of the distribution of the sample mean [PS Theorem 3.1] and the higher order asymptotic bias and variance of the KBB variance estimator [PS Theorem 3.2]. Moreover, [PS Corollaries 3.1 and 3.2], the KBB variance estimator possesses a favourable higher order bias property, a property noted elsewhere for consistent variance estimators using tapered data [Brillinger (1981, p.151)], and, for a particular choice of kernel function weighting and choice of bandwidth, is optimal being asymptotically close to one based on the optimal quadratic spectral kernel [Andrews (1991, p.821)] or Bartlett-PriestleyEpanechnikov kernel [Priestley (1962, 1981, pp. 567-571), Epanechnikov (1969) and Sacks and Ylvisacker (1981)]. Here, though, rather than being applied to the original data as in PS, the KBB kernel function weighting is applied to the individual observational components of the quasi-log likelihood criterion function itself. The asymptotic validity 
of the KBB bootstrap follows from an adaptation of the general results on resampling methods for extremum estimators given in Gonçalves and White (2004).

Myriad variants for dependent data of the bootstrap method proposed in the landmark article Efron (1979) also make use of the standard " $m$ out of $n$ " nonparametric bootstrap, but, in contrast to KBB, applied to "blocks" of the original data. See, inter alia, the moving blocks bootstrap (MBB) [Künsch (1989), Liu and Singh (1992)], the circular block bootstrap [Politis and Romano (1992a)], the stationary bootstrap [Politis and Romano (1994)], the external bootstrap for $m$-dependent data [Shi and Shao (1988)], the tapered block bootstrap (TBB) [Paparoditis and Politis (2001)] and the extended tapered block bootstrap (ETBB) [Shao (2010a)]. Whereas the block length of these other methods is typically a declining fraction of sample size, the implicit KBB block length is dictated by the support of the kernel function and, thus, with unbounded support as in the optimal case, would be the sample size itself. Non-block bootstrap methods for dependent data are the wild dependent bootstrap [Shao (2010b)], the frequency domain bootstrap [Hurvich and Zeger (1987), see also Hidalgo (2003)], and its generalization the transformationbased bootstrap [Lahiri (2003)], the autoregressive sieve bootstrap [Bühlmann (1997)], and the MA-sieve bootstrap of McMurry and Politis (2018). For further details on some of these methods, see, e.g., Kreiss and Paparoditis (2011) and the monographs Shao and $\mathrm{Tu}$ (1995) and Lahiri (2003).

When the object of inference is the stochastic process mean, the KBB method bears comparison with the tapered block bootstrap (TBB) of Paparoditis and Politis (2001). Indeed, KBB may be regarded as a generalisation and extension of TBB. TBB is also based on a reweighted sample of the observations but with weight function with bounded support and, so, whereas each KBB data point is in general a transformation of all original sample data, those of TBB use a fixed block size and, implicitly thereby, a fixed number of data points. More generally then, the TBB weight function class is a special case of that of $\mathrm{KBB}$ but is more restrictive; a detailed comparison of $\mathrm{KBB}$ and TBB is provided in PS Section 4.1. TBB is extended in Paparoditis and Politis (2002) to approximately linear statistics but differs from the KBB method introduced here for quasi-maximum likelihood estimation. In contradistinction, the extended tapered block bootstrap (ETBB) [Shao (2010a)] tapers the random weights in the bootstrapped empirical distribution rather than blocks or functions of the data. In addition to establishing the convergence of the ETBB distribution to its asymptotic counterpart, expressions for bias and the variance expansions of the ETBB variance estimator in the smooth function model are obtained in Shao (2010a) which share similar properties to those of TBB in this regard. Although 
TBB and ETBB coincide in the sample mean case, they do not in general. Investigation of an extended version of KBB similar to ETBB is left for future research.

Some considerable research has been devoted to examining the possibility of the bootstrap providing asymptotic refinements; see, e.g., the monograph Hall (1992). A basic requirement is that the statistic of interest should be asymptotically pivotal; see, e.g., Horowitz (2001). Götze and Künsch (1996) consider the smooth function model with strong mixing data and show that to obtain asymptotic refinements the estimator and its block bootstrap counterpart must be standardized appropriately. To be more precise, the standardization requires that the bias of the $\mathrm{HAC}$ variance matrix estimator should approach zero at a fast enough rate; see also Davidson and Hall (1993). Lahiri (1996) obtains similar results for the least squares estimator in the linear regression model with independence between errors and regressors. For generalised method of moments (GMM), Hall and Horowitz (1996), also see Nze and Doukhan (2004, section 2.3, 999-1001), shows that the non-overlapping block bootstrap provides asymptotic refinements for both $t$ statistics of parametric restrictions and the Hansen (1982) J-statistic for overidentifying moment restrictions after their multiplication by suitable correction factors; also see Andrews (2002, 2004). For GMM-based estimation of an instrumental variable linear regression model, Inoue and Shintani (2006) demonstrate the moving block bootstrap yields asymptotic refinements for a studentized $t$-statistic for a linear combination of the parameter vector and the distribution of the Hansen (1982) J-statistic. Since the first draft of the paper was written and after its original submission, we became aware of the recent paper La Vecchia et al. (2020) which develops a fast bootstrap method in the GMM framework similar to KBB explored here for the construction of a confidence region for the full parameter vector. Their construction of a confidence region inverts the non-rejection region of a test based on GEL estimation [Smith (2011)] utilising an asymptotically pivotal Hansen (1982) J-statistic with bootstrap critical values. The La Vecchia et al. (2020) procedure is fast not requiring parameter estimation at each bootstrap replication and achieves an asymptotic refinement.

The paper is organized as follows. After outlining some preliminaries Section 2 introduces KBB and reviews the results in PS. Section 3 demonstrates how KBB can be applied in the quasi-maximum likelihood framework and, in particular, details the consistency of the KBB estimator and its asymptotic validity for quasi-maximum likelihood. KBB methods are described appropriate for the construction of confidence regions for smooth functions of the parameter vector. Alternative KBB procedures for the full parameter vector are presented from which conservative confidence regions for smooth functions of 
the parameter vector may be obtained using the "projection" method. Section 4 reports a Monte Carlo study on the performance of KBB for the mean regression model. Finally section 5 concludes. Proofs of the results in the main text are provided in Appendix B with intermediate results required for their proofs given in Appendix A.

\section{Kernel Block Bootstrap}

To introduce the kernel block bootstrap (KBB) method, consider a sample of $T$ observations, $z_{1}, \ldots, z_{T}$, on the strictly stationary real valued $d_{z}$-dimensional vector sequence $\left\{z_{t}, t \in \mathbb{Z}\right\}$ with unknown mean $\mu=\mathrm{E}\left[z_{t}\right]$ and autocovariance sequence $R(s)=$ $\mathrm{E}\left[\left(z_{t}-\mu\right)\left(z_{t+s}-\mu\right)^{\prime}\right],(s=0, \pm 1, \ldots)$. Under suitable conditions, see Ibragimov and Linnik (1971, Theorem 18.5.3, pp. 346, 347), the limiting distribution of the sample mean $\bar{z}=$ $\sum_{t=1}^{T} z_{t} / T$ is described by $T^{1 / 2}(\bar{z}-\mu) \stackrel{d}{\rightarrow} N\left(0, \Sigma_{\infty}\right)$, where $\Sigma_{\infty}=\lim _{T \rightarrow \infty} \operatorname{var}\left[T^{1 / 2} \bar{z}\right]=$ $\sum_{s=-\infty}^{\infty} R(s)$.

Let $k_{j}=\int_{-\infty}^{\infty} k(x)^{j} d x$ with sample counterpart $\hat{k}_{j}=\sum_{s=1-T}^{T-1} k\left(s / S_{T}\right)^{j} / S_{T},(j=1,2)$, where $k(\cdot)$ denotes a suitable kernel function. The KBB approximation to the distribution of the sample mean $\bar{z}$ randomly samples the kernel-weighted centred observations

$$
z_{t T}=\frac{1}{\left(\hat{k}_{2} S_{T}\right)^{1 / 2}} \sum_{r=t-T}^{t-1} k\left(\frac{r}{S_{T}}\right)\left(z_{t-r}-\bar{z}\right),(t=1, \ldots, T),
$$

where $S_{T}$ is a bandwidth parameter.

REMARK 2.1. The definition of $z_{t T}$ (2.1) rescales that in Kitamura and Stutzer (1997) and Smith $(1997,2011)$ by $\left(S_{T} / \hat{k}_{2}\right)^{1 / 2}$ with $k_{2}$ replaced without loss by $\hat{k}_{2}$, see PS Corollary K.2, p.31.

Let $\bar{z}_{T}=T^{-1} \sum_{t=1}^{T} z_{t T}$ denote the sample mean of $z_{t T},(t=1, \ldots, T)$. Under appropriate conditions, $\bar{z}_{T} \stackrel{p}{\rightarrow} 0$ and $\Sigma_{\infty}^{-1 / 2}\left(T / S_{T}\right)^{1 / 2} \bar{z}_{T} \stackrel{d}{\rightarrow} N\left(0, I_{d_{z}}\right)$; see, e.g., Smith (2011, Lemmas A.1 and A.2, pp.1217-19). Moreover, the KBB variance estimator, defined in standard random sampling outer product form,

$$
\hat{\Sigma}_{\mathrm{KBB}}=T^{-1} \sum_{t=1}^{T}\left(z_{t T}-\bar{z}_{T}\right)\left(z_{t T}-\bar{z}_{T}\right)^{\prime} \stackrel{p}{\rightarrow} \Sigma_{\infty} ;
$$

and is thus an automatically positive semidefinite heteroskedastic and autocorrelation consistent (HAC) variance estimator; see Smith (2011, Lemma A.3, p.1219). 
KBB applies the standard " $m$ out of $n$ " non-parametric bootstrap method to the index set $\mathcal{T}_{T}=\{1, \ldots, T\}$; see Bickel and Freedman (1981). That is, the indices $t_{s}^{*}$ and, thereby, $z_{t_{s}^{*}},\left(s=1, \ldots, m_{T}\right)$, are a random sample of size $m_{T}$ drawn from, respectively, $\mathcal{T}_{T}$ and $\left\{z_{t T}\right\}_{t=1}^{T}$, where $m_{T}=\left[T / S_{T}\right]$, the integer part of $T / S_{T}$.

REMARK 2.2. The KBB sample mean $\bar{z}_{m_{T}}^{*}=\sum_{s=1}^{m_{T}} z_{t_{s}^{*} T} / m_{T}$ may be regarded as that from a random sample of size $m_{T}$ taken from the blocks $\mathcal{B}_{t}=\left\{k\left\{(t-r) / S_{T}\right\}\left(z_{r}-\right.\right.$ $\left.\bar{z}) /\left(\hat{k}_{2} S_{T}\right)^{1 / 2}\right\}_{r=1}^{T},(t=1, \ldots, T)$. See PS Remark 2.2, p.3. Note that the blocks $\left\{\mathcal{B}_{t}\right\}_{t=1}^{T}$ are overlapping and, if the kernel function $k(\cdot)$ has unbounded support, the block length is $T$.

Let $\mathcal{P}_{\omega}^{*}$ denote the bootstrap probability measure conditional on $\left\{z_{t T}\right\}_{t=1}^{T}$ (or, equivalently, the observational data $\left\{z_{t}\right\}_{t=1}^{T}$ ) with $\mathrm{E}^{*}$ and var* the corresponding conditional expectation and variance respectively. Under suitable regularity conditions, see PS Assumptions 3.1-3.3, pp.3-4, the bootstrap distribution of the scaled and centred KBB sample mean $m_{T}^{1 / 2}\left(\bar{z}_{m_{T}}^{*}-\bar{z}_{T}\right)$ converges uniformly to that of $T^{1 / 2}(\bar{z}-\mu)$, i.e.,

$$
\sup _{x \in \mathcal{R}}\left|\mathcal{P}_{\omega}^{*}\left\{m_{T}^{1 / 2}\left(\bar{z}_{m_{T}}^{*}-\bar{z}_{T}\right) / k_{1} \leq x\right\}-\mathcal{P}\left\{T^{1 / 2}(\bar{z}-\mu) \leq x\right\}\right| \rightarrow 0, \text { prob- } \mathcal{P}
$$

see PS Theorem 3.1, p.5.

Given the stricter additional requirement PS Assumption 3.4, p.5, PS Theorem 3.2, p.6, provides higher order results on moments of the KBB variance estimator $\hat{\Sigma}_{\mathrm{KBB}}(2.2)$. Let $k_{(q)}^{*}=\lim _{y \rightarrow 0}\left\{1-k^{*}(y)\right\} /|y|^{q}$, where the induced self-convolution kernel $k^{*}(y)=$ $\int_{-\infty}^{\infty} k(x-y) k(x) d x / k_{2}$. Define

$$
\operatorname{MSE}\left(T / S_{T}, \hat{\Sigma}_{\mathrm{KBB}}, W_{T}\right)=\left(T / S_{T}\right) \mathrm{E}\left[\operatorname{vec}\left(\hat{\Sigma}_{\mathrm{KBB}}-J_{T}\right)^{\prime} W_{T} v e c\left(\hat{\Sigma}_{\mathrm{KBB}}-J_{T}\right)\right]
$$

where $W_{T}$ is a positive semi-definite weight matrix and $J_{T}=\sum_{s=1-T}^{T-1}(1-|s| / T) R(s)$. Let $K_{p p}$ denote the $p^{2} \times p^{2}$ commutation matrix $\sum_{i=1}^{p} \sum_{j=1}^{p} e_{i} e_{j}^{\prime} \otimes e_{j} e_{i}^{\prime}$, where $e_{i}$ is the $i$ th elementary vector, $(i=1, \ldots, p)$, (Magnus and Neudecker, 1979, Definition 3.1, p.383).

BiAs. $\mathrm{E}\left[\hat{\Sigma}_{\mathrm{KBв}}\right]=J_{T}+S_{T}^{-2}\left(\Gamma_{k^{*}}+o(1)\right)+U_{T}$, where $\Gamma_{k^{*}}=-k_{(2)}^{*} \sum_{s=-\infty}^{\infty}|s|^{2} R(s)$ and $U_{T}=O\left(\left(S_{T} / T\right)^{b-1 / 2}\right)+o\left(1 / S_{T}^{2}\right)+O\left(S_{T}^{b-2} / T^{b}\right)+O\left(S_{T} / T\right)+O\left(\left(S_{T} / T\right)^{3 / 2-\varepsilon}\right)$ with $b>1$ and $\varepsilon \in(0,1 / 2)$;

VARIANCE. if $S_{T}^{5} / T \rightarrow \gamma \in(0, \infty)$, then $\left(T / S_{T}\right) \operatorname{var}\left[\hat{\Sigma}_{\mathrm{KBB}}\right]=\Delta_{k^{*}}+o(1)$, where $\Delta_{k^{*}}=$ $\left(I_{p p}+K_{p p}\right)\left(\Sigma_{\infty} \otimes \Sigma_{\infty}\right) \int_{-\infty}^{\infty} k^{*}(y)^{2} d y$ 
Mean Squared Error. if $S_{T}^{5} / T \rightarrow \gamma \in(0, \infty)$, then $\operatorname{MSE}\left(T / S_{T}, \hat{\Sigma}_{\mathrm{KB}}, W_{T}\right)=$ $\operatorname{tr}\left(W\left(I_{p p}+K_{p p}\right)\left(\Sigma_{\infty} \otimes \Sigma_{\infty}\right)\right) \int_{-\infty}^{\infty} k^{*}(y)^{2} d y+\operatorname{vec}\left(\Gamma_{k^{*}}\right)^{\prime} W v e c\left(\Gamma_{k^{*}}\right) / \gamma+o(1)$.

Remark 2.3. The bias and variance results are similar to Parzen (1957, Theorems 5A and 5B, pp.339-340) and Andrews (1991, Proposition 1, p.825), when the Parzen exponent $q$ equals 2 . The KBB bias, cf. the tapered block bootstrap (TBB), is $O\left(1 / S_{T}^{2}\right)$, an improvement on $O\left(1 / S_{T}\right)$ for the moving block bootstrap (MBB). The expression $\operatorname{MSE}\left(T / S_{T}, \hat{\Sigma}_{\mathrm{KBB}}, W_{T}\right)$ is identical to that for the mean squared error of the Parzen (1957) estimator based on the induced self-convolution kernel $k^{*}(y)$.

Optimality results for the estimation of $\Sigma_{\infty}$ are an immediate consequence of PS Theorem 3.2, p.6, and the theoretical results of Andrews (1991) for the Parzen (1957) estimator. Consider the kernel function

$$
k(x)=\left(\frac{5 \pi}{8}\right)^{1 / 2} \frac{1}{x} J_{1}\left(\frac{6 \pi x}{5}\right) \text { if } x \neq 0 \text { and }\left(\frac{5 \pi}{8}\right)^{1 / 2} \frac{3 \pi}{5} \text { if } x=0 ;
$$

here $J_{v}(z)=\sum_{k=0}^{\infty}(-1)^{k}(z / 2)^{2 k+v} /\{\Gamma(k+1) \Gamma(k+2)\}$, a Bessel function of the first kind (Gradshteyn and Ryzhik, 1980, 8.402, p.951) with $\Gamma(\cdot)$ the gamma function. Smith (2011, Example 2.3, p.1204) shows that the quadratic spectral (QS) kernel $k_{\mathrm{QS}}^{*}(y)$ is the induced self-convolution kernel $k^{*}(y)$ associated with the kernel $k(x)(2.4)$, where the QS kernel

$$
k_{\mathrm{QS}}^{*}(y)=\frac{3}{(a y)^{2}}\left(\frac{\sin a y}{a y}-\cos a y\right), a=6 \pi / 5,
$$

REMARK 2.4. The QS kernel $k_{\mathrm{QS}}^{*}(y)(2.5)$ is well-known to possess optimality properties, e.g., for the estimation of spectral densities (Priestley, 1962; 1981, pp. 567-571) and probability densities (Epanechnikov, 1969, Sacks and Ylvisacker, 1981).

PS Corollary 3.1, p.7, establishes an optimality result for the KBB variance estimator $\hat{\Sigma}_{\text {Квв }}\left(S_{T}\right)(2.2)$ computed with the kernel function (2.4) which is denoted as $\tilde{\Sigma}_{\text {Квв }}\left(S_{T}\right)$. For sensible comparisons, the requisite bandwidth parameter is $S_{T k^{*}}=S_{T} / \int_{-\infty}^{\infty} k^{*}(y)^{2} d y$, see Andrews (1991, (4.1), p.829), if the respective asymptotic variances scaled by $T / S_{T}$ are to coincide; see Andrews (1991, p.829). Then, for any bandwidth sequence $S_{T}$ such that $S_{T} \rightarrow \infty$ and $S_{T}^{5} / T \rightarrow \gamma \in(0, \infty), \lim _{T \rightarrow \infty} \operatorname{MSE}\left(T / S_{T}, \hat{\Sigma}_{\mathrm{KBB}}, W_{T}\right)-\operatorname{MSE}\left(T / S_{T}, \tilde{\Sigma}_{\mathrm{KBB}}, W_{T}\right) \geq$ 0 with strict inequality if $k^{*}(y) \neq k_{\mathrm{QS}}^{*}(y)$ with positive Lebesgue measure; see PS Corollary 3.1, p.7. 
The bandwidth $S_{T}^{*}=\left[\operatorname{4vec}\left(\Gamma_{k^{*}}\right)^{\prime} W \operatorname{vec}\left(\Gamma_{k^{*}}\right) / \operatorname{tr}\left(W\left(I_{p p}+K_{p p}\right)\left(\Sigma_{\infty} \otimes \Sigma_{\infty}\right)\right) \int_{-\infty}^{\infty} k^{*}(y)^{2} d y\right]^{1 / 5} T^{1 / 5}$ is also optimal in the following sense. For any bandwidth sequence $S_{T}$ such that $S_{T} \rightarrow \infty$ and $S_{T}^{5} / T \rightarrow \gamma \in(0, \infty), \lim _{T \rightarrow \infty} \operatorname{MSE}\left(T^{4 / 5}, \hat{\Sigma}_{\text {Квв }}\left(S_{T}\right), W_{T}\right)-\operatorname{MSE}\left(T^{4 / 5}, \hat{\Sigma}_{\mathrm{KBв}}\left(S_{T}^{*}\right), W_{T}\right) \geq$ 0 with strict inequality unless $S_{T}=S_{T}^{*}+o\left(1 / T^{1 / 5}\right)$; see PS Corollary 3.2, p.7.

\section{Quasi-Maximum LiKelihood}

This section applies the KBB method briefly outlined above to parameter estimation in the quasi-maximum likelihood (QML) setting. In particular, under the regularity conditions detailed below, KBB may be used to construct hypothesis tests and confidence intervals or regions for smooth functions of the parameter vector. The proofs of the results basically rely on verifying a number of the conditions required for several general lemmas established in Gonçalves and White (2004) on resampling methods for extremum estimators. Indeed, although the focus of Gonçalves and White (2004) is MBB, the results therein also apply to other block bootstrap schemes such as KBB.

To describe the set-up, let the $d_{z}$-vectors $z_{t},(t=1, \ldots, T)$, denote a realisation from the stationary and strong mixing stochastic process $\left\{z_{t}\right\}_{t=1}^{\infty}$. The $d_{\theta}$-vector $\theta$ of parameters is of interest where $\theta \in \Theta$ with the compact parameter space $\Theta \subseteq \mathcal{R}^{d_{\theta}}$. Consider the $\log$-density $\mathcal{L}_{t}(\theta)=\log f\left(z_{t} ; \theta\right)$ and its expectation $\mathcal{L}(\theta)=\mathrm{E}\left[\mathcal{L}_{t}(\theta)\right]$. The true value $\theta_{0}$ of $\theta$ is defined by

$$
\theta_{0}=\arg \max _{\theta \in \Theta} \mathcal{L}(\theta)
$$

with, analogously, the QML estimator $\hat{\theta}$ of $\theta_{0}$

$$
\hat{\theta}=\arg \max _{\theta \in \Theta} \overline{\mathcal{L}}(\theta)
$$

where the sample mean $\overline{\mathcal{L}}(\theta)=\sum_{t=1}^{T} \mathcal{L}_{t}(\theta) / T$.

The KBB method for QML makes use of the kernel smoothed log density function

$$
\mathcal{L}_{t T}(\theta)=\frac{1}{\left(\hat{k}_{2} S_{T}\right)^{1 / 2}} \sum_{r=t-T}^{t-1} k\left(\frac{r}{S_{T}}\right) \mathcal{L}_{t-r}(\theta),(t=1, \ldots, T),
$$

cf. (2.1). As in Section 2, the indices $t_{s}^{*}$ and the consequent bootstrap sample $\mathcal{L}_{t_{s}^{*} T}(\theta)$, $\left(s=1, \ldots, m_{T}\right)$, denote random samples of size $m_{T}$ drawn with replacement from the index set $\mathcal{T}_{T}=\{1, \ldots, T\}$ and the bootstrap sample space $\left\{\mathcal{L}_{t T}(\theta)\right\}_{t=1}^{T}$, where $m_{T}=\left[T / S_{T}\right]$ is the integer part of $T / S_{T}$. The bootstrap QML estimator $\hat{\theta}^{*}$ is then defined by

$$
\hat{\theta}^{*}=\arg \max _{\theta \in \Theta} \overline{\mathcal{L}}_{m_{T}}^{*}(\theta)
$$


where the bootstrap sample mean $\overline{\mathcal{L}}_{m_{T}}^{*}(\theta)=\sum_{s=1}^{m_{T}} \mathcal{L}_{t_{s}^{*} T}(\theta) / m_{T}$.

REMARK 3.1. Note that, because $\mathrm{E}\left[\partial \mathcal{L}_{t}\left(\theta_{0}\right) / \partial \theta\right]=0$, it is unnecessary to centre $\mathcal{L}_{t}(\theta)$, $(t=1, \ldots, T)$, at $\overline{\mathcal{L}}(\theta) ;$ cf. $(2.1)$.

The following conditions are imposed to establish the consistency of the bootstrap estimator $\hat{\theta}^{*}$ for $\theta_{0}$. Let $f_{t}(\theta)=f\left(z_{t} ; \theta\right),(t=1,2, \ldots)$.

Assumption 3.1 (a) $(\Omega, \mathcal{F}, \mathcal{P})$ is a complete probability space; (b) the finite $d_{z}$-dimensional stochastic process $z_{t}: \Omega \longmapsto \mathcal{R}^{d_{z}},(t=1,2, \ldots)$, is stationary and strong mixing with mixing numbers of size $-v /(v-1)$ for some $v>1$ and is measurable for all $t,(t=1,2, \ldots)$.

Assumption 3.2 (a) $f: \mathcal{R}^{d_{z}} \times \Theta \longmapsto \mathcal{R}^{+}$is $\mathcal{F}$-measurable for each $\theta \in \Theta, \Theta$ a compact subset of $\mathcal{R}^{d_{\theta}}$; (b) $f_{t}(\cdot): \Theta \longmapsto \mathcal{R}^{+}$is continuous on $\Theta$ a.s. $\mathcal{P}$; (c) $\theta_{0} \in \Theta$ is the unique maximizer of $\mathrm{E}\left[\log f_{t}(\theta)\right], \mathrm{E}\left[\sup _{\theta \in \Theta}\left|\log f_{t}(\theta)\right|^{\alpha}\right]<\infty$ for some $\alpha>v$; (d) $\log f_{t}(\theta)$ is global Lipschitz continuous on $\Theta$, i.e., for all $\theta, \theta^{0} \in \Theta,\left|\log f_{t}(\theta)-\log f_{t}\left(\theta^{0}\right)\right| \leq$ $L_{t}\left\|\theta-\theta^{0}\right\|$ a.s. $\mathcal{P}$ and $\sup _{T} \mathrm{E}\left[\sum_{t=1}^{T} L_{t} / T\right]<\infty$;

Let $\mathbb{I}(x \geq 0)$ denote the indicator function, i.e., $\mathbb{I}(A)=1$ if $A$ true and 0 otherwise.

Assumption 3.3 (a) $S_{T} \rightarrow \infty$ and $S_{T}=o\left(T^{\frac{1}{2}}\right)$; (b) $k(\cdot): \mathcal{R} \longmapsto\left[-k_{\max }, k_{\max }\right], k_{\max }<$ $\infty, k(0) \neq 0, k_{1} \neq 0$, and is continuous at 0 and almost everywhere; (c) $\int_{-\infty}^{\infty} \bar{k}(x) d x<\infty$ where $\bar{k}(x)=\mathbb{I}(x \geq 0) \sup _{y \geq x}|k(y)|+\mathbb{I}(x<0) \sup _{y \leq x}|k(y)| ;(\mathbf{d})|K(\lambda)| \geq 0$ for all $\lambda \in \mathcal{R}$, where $K(\lambda)=(2 \pi)^{-1} \int k(x) \exp (-i x \lambda) d x$.

Theorem 3.1. Let Assumptions 3.1-3.3 hold. Then, if $T^{1 / \alpha} / m_{T} \rightarrow 0$, (a) $\hat{\theta}-\theta_{0} \rightarrow 0$, prob- $\mathcal{P}$; (b) $\hat{\theta}^{*}-\hat{\theta} \rightarrow 0$, prob- $\mathcal{P}_{\omega}^{*}$, prob- $\mathcal{P}$.

To prove consistency of the KBB distribution requires a strengthening of the above assumptions.

Assumption 3.4 (a) $(\Omega, \mathcal{F}, \mathcal{P})$ is a complete probability space; (b) the finite $d_{z}$-dimensional stochastic process $z_{t}: \Omega \longmapsto \mathcal{R}^{d_{z}},(t=1,2, \ldots)$, is stationary and strong mixing with mixing numbers of size $-3 v /(v-1)$ for some $v>1$ and is measurable for all $t,(t=1,2, \ldots)$.

Assumption 3.5 (a) $f: \mathcal{R}^{d_{z}} \times \Theta \longmapsto \mathcal{R}^{+}$is $\mathcal{F}$-measurable for each $\theta \in \Theta, \Theta$ a compact subset of $\mathcal{R}^{d_{\theta}}$; (b) $f_{t}(\cdot): \Theta \longmapsto \mathcal{R}^{+}$is continuously differentiable of order 2 on $\Theta$ a.s.-P, $(t=1,2, \ldots)$; (c) $\theta_{0} \in \operatorname{int}(\Theta)$ is the unique maximizer of $\mathrm{E}\left[\log f_{t}(\theta)\right]$. 
Define $A(\theta)=\mathrm{E}\left[\partial^{2} \mathcal{L}_{t}(\theta) / \partial \theta \partial \theta^{\prime}\right]$ and $B(\theta)=\lim _{T \rightarrow \infty} \operatorname{var}\left[T^{1 / 2} \partial \overline{\mathcal{L}}(\theta) / \partial \theta\right]$.

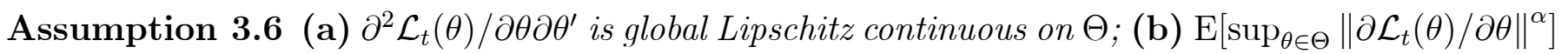
$<\infty$ and $\mathrm{E}\left[\sup _{\theta \in \Theta}\left\|\partial^{2} \mathcal{L}_{t}(\theta) / \partial \theta \partial \theta^{\prime}\right\|^{\alpha}\right]<\infty$ for some $\alpha>\max [4 v, 1 / \eta] ;$ (c) $A_{0}=A\left(\theta_{0}\right)$ is non-singular and $B_{0}=\lim _{T \rightarrow \infty} \operatorname{var}\left[T^{1 / 2} \partial \overline{\mathcal{L}}\left(\theta_{0}\right) / \partial \theta\right]$ is positive definite.

REMARK 3.2. Assumption 3.6(b) obviates the condition $T^{\alpha} / m_{T} \rightarrow 0$ of Theorem 3.1 required by the bootstrap pointwise WLLN Lemma A.2 in Appendix A.

Under these regularity conditions,

$$
B_{0}^{-1 / 2} A_{0} T^{1 / 2}\left(\hat{\theta}-\theta_{0}\right) \stackrel{d}{\rightarrow} N\left(0, I_{d_{\theta}}\right)
$$

see the Proof of Theorem 3.2. Moreover,

Theorem 3.2. Suppose Assumptions 3.2-3.6 are satisfied. Then, if $S_{T} \rightarrow \infty$ and $S_{T}=O\left(T^{\frac{1}{2}-\eta}\right)$ with $0<\eta<\frac{1}{2}$

$$
\sup _{x \in \mathcal{R}^{d_{\theta}}}\left|\mathcal{P}_{\omega}^{*}\left\{T^{1 / 2}\left(\hat{\theta}^{*}-\hat{\theta}\right) / k^{1 / 2} \leq x\right\}-\mathcal{P}\left\{T^{1 / 2}\left(\hat{\theta}-\theta_{0}\right) \leq x\right\}\right| \rightarrow 0, \text { prob- } \mathcal{P},
$$

where $k=k_{2} / k_{1}^{2}$.

REMARK 3.3. The factor $k$ may be replaced without loss by $\hat{k}=\hat{k}_{2} / \hat{k}_{1}^{2}$, see PS Corollary K.2, p.31. Cf. Remark 2.1.

Let $\overline{\mathcal{L}}_{T}(\theta)=\sum_{t=1}^{T} \mathcal{L}_{t T}(\theta) / T$. An alternative less computationally intensive centred fast bootstrap may be based on the next result. Cf. the fast subsampling procedure of Hong and Scaillet (2006); see also Camponovo et al. (2012).

Corollary 3.1. Under the conditions of Theorem 3.2, if $S_{T} \rightarrow \infty$ and $S_{T}=$ $O\left(T^{\frac{1}{2}-\eta}\right)$ with $0<\eta<\frac{1}{2}$

$\sup _{x \in \mathcal{R}^{d_{\theta}}}\left|\mathcal{P}_{\omega}^{*}\left\{-\left[\frac{\partial^{2} \overline{\mathcal{L}}_{m_{T}}^{*}(\hat{\theta})}{\partial \theta \partial \theta^{\prime}}\right]^{-1} T^{1 / 2}\left(\frac{\partial \overline{\mathcal{L}}_{m_{T}}^{*}(\hat{\theta})}{\partial \theta}-\frac{\partial \overline{\mathcal{L}}_{T}(\hat{\theta})}{\partial \theta}\right) / k^{1 / 2} \leq x\right\}-\mathcal{P}\left\{T^{1 / 2}\left(\hat{\theta}-\theta_{0}\right) \leq x\right\}\right| \rightarrow 0$, prob- $\mathcal{P}$ where $k=k_{2} / k_{1}^{2}$.

Remark 3.4. From the bootstrap UWL Lemma A.1 in Appendix A, suitably adapted, the matrix $\partial^{2} \overline{\mathcal{L}}_{T}(\hat{\theta}) / \partial \theta \partial \theta^{\prime}$ may substitute for $\partial^{2} \overline{\mathcal{L}}_{m_{T}}^{*}(\hat{\theta}) / \partial \theta \partial \theta^{\prime}$ in Corollary 3.1, 
viz. $\sup _{\theta \in \Theta}\left(k_{2} / S_{T}\right)^{1 / 2}\left\|\partial^{2} \overline{\mathcal{L}}_{m_{T}}^{*}(\theta) / \partial \theta \partial \theta^{\prime}-\partial^{2} \overline{\mathcal{L}}_{T}(\theta) / \partial \theta \partial \theta^{\prime}\right\| \rightarrow 0$, prob- $\mathcal{P}_{\omega}^{*}$, prob- $\mathcal{P}$. A similar argument together with the UWLs $\sup _{\theta \in \Theta}\left\|\left(k_{2} / S_{T}\right)^{1 / 2} \partial^{2} \overline{\mathcal{L}}_{T}(\theta) / \partial \theta \partial \theta^{\prime}-k_{1} A(\theta)\right\| \rightarrow$ 0 , prob- $\mathcal{P}$, and $\sup _{\theta \in \Theta}\left\|\partial^{2} \overline{\mathcal{L}}(\theta) / \partial \theta \partial \theta^{\prime}-A(\theta)\right\| \rightarrow 0$, prob- $\mathcal{P}$, yields

$$
\sup _{x \in \mathcal{R}^{d_{\theta}}}\left|\mathcal{P}_{\omega}^{*}\left\{-\left[\frac{\partial^{2} \overline{\mathcal{L}}(\hat{\theta})}{\partial \theta \partial \theta^{\prime}}\right]^{-1} m_{T}^{1 / 2}\left(\frac{\partial \overline{\mathcal{L}}_{m_{T}}^{*}(\hat{\theta})}{\partial \theta}-\frac{\partial \overline{\mathcal{L}}_{T}(\hat{\theta})}{\partial \theta}\right) \leq x\right\}-\mathcal{P}\left\{T^{1 / 2}\left(\hat{\theta}-\theta_{0}\right) \leq x\right\}\right| \rightarrow 0, \text { prob-} \mathcal{P},
$$

similarly to Paparoditis and Politis (2002, Theorem 2.2, p.135) for the QML implementation of TBB expressed in terms of the influence function corresponding to the QML criterion $\overline{\mathcal{L}}(\theta)$, viz.,

$$
I F\left(z, \hat{F}_{T}\right)=-\left[\frac{\partial^{2} \overline{\mathcal{L}}(\hat{\theta})}{\partial \theta \partial \theta^{\prime}}\right]^{-1}\left(\frac{\partial \mathcal{L}_{t}(\hat{\theta})}{\partial \theta}-\frac{\partial \overline{\mathcal{L}}(\hat{\theta})}{\partial \theta}\right),(t=1, \ldots, T)
$$

noting that $\partial \overline{\mathcal{L}}(\hat{\theta}) / \partial \theta=0$.

REMARK 3.5. As noted in Remark 3.4, $\partial^{2} \overline{\mathcal{L}}(\hat{\theta}) / \partial \theta \partial \theta^{\prime}$ may be replaced by $\left(k / S_{T}\right)^{1 / 2} \partial^{2} \overline{\mathcal{L}}_{T}(\hat{\theta}) / \partial \theta \partial \theta^{\prime}$. Hence,

$$
\sup _{x \in \mathcal{R}^{d} \theta}\left|\mathcal{P}_{\omega}^{*}\left\{-\left[\frac{\partial^{2} \overline{\mathcal{L}}_{T}(\hat{\theta})}{\partial \theta \partial \theta^{\prime}}\right]^{-1} T^{1 / 2}\left(\frac{\partial \overline{\mathcal{L}}_{m_{T}}^{*}(\hat{\theta})}{\partial \theta}-\frac{\partial \overline{\mathcal{L}}_{T}(\hat{\theta})}{\partial \theta}\right) / k^{1 / 2} \leq x\right\}-\mathcal{P}\left\{T^{1 / 2}\left(\hat{\theta}-\theta_{0}\right) \leq x\right\}\right| \rightarrow 0, \text { prob-P. }
$$

REMARK 3.6. It follows from the first order condition $\partial \overline{\mathcal{L}}(\hat{\theta}) / \partial \theta=0$ for $\hat{\theta}$ that the term $\partial \overline{\mathcal{L}}_{T}(\hat{\theta}) / \partial \theta$ in Corollary 3.1 and Remarks 3.4 and 3.5 may be omitted. The corresponding uncentred bootstrap from Remark 3.4 is the KBB version of Gonçalves and White (2004, Corollary 2.1, p.203) for MBB applied to the one-step QML estimator; also see Davidson and MacKinnon (1999).

REMARK 3.7. The KBB variance estimator for the large sample variance matrix $A_{0}^{-1} B_{0} A_{0}^{-1}$ of the QML estimator $\hat{\theta}$ ( or $\hat{\theta}^{*}$ ) is given by the outer product form

$$
\frac{1}{T}\left[\frac{\partial^{2} \overline{\mathcal{L}}_{T}(\hat{\theta})}{\partial \theta \partial \theta^{\prime}}\right]^{-1} \sum_{t=1}^{T} \frac{\partial \mathcal{L}_{t T}(\hat{\theta})}{\partial \theta} \frac{\partial \mathcal{L}_{t T}(\hat{\theta})}{\partial \theta^{\prime}}\left[\frac{\partial^{2} \overline{\mathcal{L}}_{T}(\hat{\theta})}{\partial \theta \partial \theta^{\prime}}\right]^{-1}
$$

or

$$
\frac{1}{T}\left[\frac{\partial^{2} \overline{\mathcal{L}}_{T}(\hat{\theta})}{\partial \theta \partial \theta^{\prime}}\right]^{-1} \sum_{t=1}^{T}\left(\frac{\partial \mathcal{L}_{t T}(\hat{\theta})}{\partial \theta}-\frac{\partial \mathcal{L}_{T}(\hat{\theta})}{\partial \theta}\right)\left(\frac{\partial \mathcal{L}_{t T}(\hat{\theta})}{\partial \theta}-\frac{\partial \mathcal{L}_{T}(\hat{\theta})}{\partial \theta}\right)^{\prime}\left[\frac{\partial^{2} \overline{\mathcal{L}}_{T}(\hat{\theta})}{\partial \theta \partial \theta^{\prime}}\right]^{-1}
$$

Cf. (2.2); see PS (2.2), p.2, and Smith (2005, Theorem 2.1, p.165, and 2011, Lemma A.3, p.1219). 
Other statistics may be defined if a confidence region for the full vector $\theta_{0}$ is of primary interest; conservative confidence regions for a smooth function of the parameter vector may be obtained using the "projection" method, see, e.g., Dufour (2003, Section 6.2, 791-792). In particular, the likelihood ratio (LR) statistic, although not asymptotically pivotal here, may still prove to be useful for inference although any bootstrap approximation to its distribution would not provide an asymptotic refinement. However, the LR statistic has a number of advantages: it does not require explicit estimation of the long run variance matrix $B_{0}$, it is transformation equivariant and is not computationally complex.

Let $\mathcal{M}_{d_{\theta}}(\cdot, \lambda)$ denote the c.d.f. of a weighted sum of $d_{\theta}$ independent chi-square distributed random variables each with one degree of freedom and each weighted by successive elements of $\lambda$ the $d_{\theta}$-vector of eigenvalues of $-B_{0} A_{0}^{-1}$; see Vuong (1989, Definition 1 , p.312). Under the regularity conditions of Theorem 3.2, the LR statistic $2 T\left(\overline{\mathcal{L}}(\hat{\theta})-\overline{\mathcal{L}}\left(\theta_{0}\right)\right)$ converges in distribution to $\mathcal{M}_{d_{\theta}}(\cdot, \lambda)$, prob- $\mathcal{P}$; see the Proof of Theorem 3.3. Moreover,

ThEOREM 3.3. Under the conditions of Theorem 3.2,

$$
\sup _{x \in \mathcal{R}^{d} \theta}\left|\mathcal{P}_{\omega}^{*}\left\{2 T\left(\overline{\mathcal{L}}_{m_{T}}^{*}\left(\hat{\theta}^{*}\right)-\overline{\mathcal{L}}_{m_{T}}^{*}(\hat{\theta})\right) /\left(k S_{T}\right)^{1 / 2} \leq x\right\}-\mathcal{P}\left\{2 T\left(\overline{\mathcal{L}}(\hat{\theta})-\overline{\mathcal{L}}\left(\theta_{0}\right)\right) \leq x\right\}\right| \rightarrow 0, \text { prob-} \mathcal{P} .
$$

REMARK 3.8. It immediately follows from the Proof of Theorem 3.3 that an asymptotically equivalent KBB Wald-type statistic $\left.-T\left(\hat{\theta}^{*}-\hat{\theta}\right)^{\prime} \partial^{2} \overline{\mathcal{L}}_{m_{T}}^{*}\left(\hat{\theta}^{*}\right) / \partial \theta \partial \theta^{\prime}\right]\left(\hat{\theta}^{*}-\right.$ $\hat{\theta}) /\left(k S_{T}\right)^{1 / 2}$ may be defined; viz.

$$
\sup _{x \in \mathcal{R}^{d_{\theta}}}\left|\mathcal{P}_{\omega}^{*}\left\{-T\left(\hat{\theta}^{*}-\hat{\theta}\right)^{\prime} \frac{\partial^{2} \overline{\mathcal{L}}_{m_{T}}^{*}\left(\hat{\theta}^{*}\right)}{\partial \theta \partial \theta^{\prime}}\left(\hat{\theta}^{*}-\hat{\theta}\right) /\left(k S_{T}\right)^{1 / 2} \leq x\right\}-\mathcal{P}\left\{2 T\left(\overline{\mathcal{L}}(\hat{\theta})-\overline{\mathcal{L}}\left(\theta_{0}\right)\right) \leq x\right\}\right| \rightarrow 0, \text { prob- } \mathcal{P} .
$$

As noted in Remarks 3.4 and 3.5, $\partial^{2} \overline{\mathcal{L}}_{T}\left(\hat{\theta}^{*}\right) / \partial \theta \partial \theta^{\prime}$ or $\left(k / S_{T}\right)^{-1 / 2} \partial^{2} \overline{\mathcal{L}}\left(\hat{\theta}^{*}\right) / \partial \theta \partial \theta^{\prime}$ may be substituted for $\partial^{2} \overline{\mathcal{L}}_{m_{T}}^{*}\left(\hat{\theta}^{*}\right) / \partial \theta \partial \theta^{\prime}$ with $\hat{\theta}$ replacing $\hat{\theta}^{*}$.

REMARK 3.9. Let $x_{\alpha}^{*}$ denote the $\alpha$-level KBB critical value from either Theorem 3.3 or Remark 3.8. A nominal $\alpha$-level confidence region for $\theta_{0}$ is provided by inversion of the non-rejection region $\left\{2 T(\overline{\mathcal{L}}(\hat{\theta})-\overline{\mathcal{L}}(\theta)) \leq x_{\alpha}^{*}\right\}$.

Of course, neither the LR nor the Wald-type statistic are asymptotically pivotal statistics. To remedy for this difficulty, first, define

$$
\hat{A}_{T}(\theta)=\left(k / S_{T}\right)^{1 / 2} \frac{\partial^{2} \overline{\mathcal{L}}_{T}(\theta)}{\partial \theta \partial \theta^{\prime}}, \hat{B}_{T}(\theta)=\frac{1}{T} \sum_{t=1}^{T}\left(\frac{\partial \mathcal{L}_{t T}(\theta)}{\partial \theta}-\frac{\partial \overline{\mathcal{L}}_{T}(\theta)}{\partial \theta}\right)\left(\frac{\partial \mathcal{L}_{t T}(\theta)}{\partial \theta}-\frac{\partial \overline{\mathcal{L}}_{T}(\theta)}{\partial \theta}\right)^{\prime} .
$$


and, secondly, let $\chi_{d_{\theta}}^{2}(\cdot)$ denote a chi-square c.d.f. with $d_{\theta}$ degrees of freedom. Since $\hat{A}_{T}(\hat{\theta}) \rightarrow A_{0}$ and $\hat{B}_{T}(\hat{\theta}) \rightarrow B_{0}$, prob- $\mathcal{P}$, the statistic $T\left(\hat{\theta}-\theta_{0}\right)^{\prime} \hat{A}_{T}(\hat{\theta}) \hat{B}_{T}(\hat{\theta})^{-1} \hat{A}_{T}(\hat{\theta})\left(\hat{\theta}-\theta_{0}\right)$ converges in distribution to $\chi_{d_{\theta}}^{2}(\cdot)$, prob- $\mathcal{P}$, and is, thus, asymptotically pivotal. The next result is then immediate; cf. Theorem 3.2.

THEOREM 3.4. Under the conditions of Theorem 3.2,

$$
\begin{aligned}
\sup _{x \in \mathcal{R}^{d} \theta} & \mathcal{P}_{\omega}^{*}\left\{T\left(\hat{\theta}^{*}-\hat{\theta}\right)^{\prime} \hat{A}_{T}(\hat{\theta}) \hat{B}_{T}(\hat{\theta})^{-1} \hat{A}_{T}(\hat{\theta})\left(\hat{\theta}^{*}-\hat{\theta}\right) / k \leq x\right\} \\
& -\mathcal{P}\left\{T\left(\hat{\theta}-\theta_{0}\right)^{\prime} \hat{A}_{T}\left(\theta_{0}\right) \hat{B}_{T}\left(\theta_{0}\right)^{-1} \hat{A}_{T}\left(\theta_{0}\right)\left(\hat{\theta}-\theta_{0}\right) \leq x\right\} \rightarrow 0, \text { prob-P. }
\end{aligned}
$$

Likewise, as $\hat{A}_{T}\left(\theta_{0}\right) T^{1 / 2}\left(\hat{\theta}-\theta_{0}\right)-T^{1 / 2} \partial \overline{\mathcal{L}}\left(\theta_{0}\right) / \partial \theta \rightarrow 0$, prob- $\mathcal{P}$, the score-type statistic $\left[\partial \overline{\mathcal{L}}\left(\theta_{0}\right) / \partial \theta^{\prime}\right] \hat{B}_{T}\left(\theta_{0}\right)^{-1}\left[\partial \overline{\mathcal{L}}\left(\theta_{0}\right) / \partial \theta\right] \stackrel{d}{\rightarrow} \chi_{d_{\theta}}^{2}(\cdot)$, prob- $\mathcal{P}$, and is asymptotically pivotal. Noting $\sup _{x \in \mathcal{R}^{d_{\theta}}}\left|\mathcal{P}_{\omega}^{*}\left\{m_{T}^{1 / 2} \partial \overline{\mathcal{L}}_{m_{T}}^{*}\left(\hat{\theta}_{0}\right) / \partial \theta \leq x\right\}-\mathcal{P}\left\{T^{1 / 2} \partial \overline{\mathcal{L}}\left(\theta_{0}\right) / \partial \theta \leq x\right\}\right| \rightarrow 0$, prob- $\mathcal{P}$, see Proof of Corollary 3.1, consider the bootstrap score-type statistic $m_{T}\left[\partial \overline{\mathcal{L}}_{m_{T}}^{*}(\hat{\theta}) / \partial \theta\right]^{\prime} \hat{B}_{T}(\hat{\theta})^{-1}\left[\partial \overline{\mathcal{L}}_{m_{T}}^{*}(\hat{\theta}) / \partial \theta\right]$

Corollary 3.3. Under the conditions of Theorem 3.2,

$$
\sup _{x \in \mathcal{R}^{d_{\theta}}}\left|\mathcal{P}_{\omega}^{*}\left\{m_{T} \frac{\partial \overline{\mathcal{L}}_{m_{T}}^{*}(\hat{\theta})}{\partial \theta^{\prime}} \hat{B}_{T}(\hat{\theta})^{-1} \frac{\partial \overline{\mathcal{L}}_{m_{T}}^{*}(\hat{\theta})}{\partial \theta} \leq x\right\}-\mathcal{P}\left\{T \frac{\partial \overline{\mathcal{L}}\left(\theta_{0}\right)}{\partial \theta} \hat{B}_{T}\left(\theta_{0}\right)^{-1} \frac{\partial \overline{\mathcal{L}}\left(\theta_{0}\right)}{\partial \theta} \leq x\right\}\right| \rightarrow 0, \text { prob-P. }
$$

REMARK 3.10. The KBB statistics in Theorem 3.4 and Corollary 3.3 potentially provide the basis for asymptotic refinements although exploration of this topic is beyond the scope of the paper. The bootstrap score-type statistic $m_{T}\left[\partial \overline{\mathcal{L}}_{m_{T}}^{*}(\hat{\theta}) / \partial \theta^{\prime}\right] \hat{B}_{T}(\hat{\theta})^{-1}\left[\partial \overline{\mathcal{L}}_{m_{T}}^{*}(\hat{\theta}) / \partial \theta\right]$ is similar to the bootstrap Hansen (1982) J-statistic $Q^{*}$ proposed in LaVecchia et al. (2020, (11), p.9) in the GMM framework except the bootstrap sample size $m_{T}=T$ and the centred score $\partial \overline{\mathcal{L}}_{m_{T}}^{*}(\hat{\theta}) / \partial \theta-\partial \overline{\mathcal{L}}_{T}(\hat{\theta}) / \partial \theta$ and the bootstrap variance estimator $\sum_{s=1}^{m_{T}}\left(\partial \mathcal{L}_{t_{s}^{*} T}(\hat{\theta}) / \partial \theta-\partial \overline{\mathcal{L}}_{T}(\hat{\theta}) / \partial \theta\right)\left(\partial \mathcal{L}_{t_{s}^{*} T}(\hat{\theta}) / \partial \theta-\partial \overline{\mathcal{L}}_{T}(\hat{\theta}) / \partial \theta\right)^{\prime} / m_{T}$ substitute for $\partial \overline{\mathcal{L}}_{m_{T}}^{*}(\hat{\theta}) / \partial \theta$ and $\hat{B}_{T}(\hat{\theta})$ respectively with the GEL estimator replacing the QML estimator $\hat{\theta}$. Note that, in the QML framework, the GEL estimator satisfies $\partial \overline{\mathcal{L}}_{T}(\theta) / \partial \theta=0$. LaVecchia et al. (2020, Theorem 2, p.14) shows that the resultant bootstrap confidence region for $\theta_{0}$ based on $Q^{*}$ provides an asymptotic refinement. However, if the optimal kernel function $k(\cdot)(2.4)$ corresponding to its self-convolution QS kernel $k_{\mathrm{QS}}^{*}(\cdot)(2.5)$ is used to smooth the log-density and resultant score, the optimal bandwidth $S_{T}^{*}=O\left(T^{-1 / 5}\right)$ is inadmissible for an asymptotic refinement since $S_{T}$ is required to be faster than $T^{-1 / 4}$ and slower than $T^{-1 / 2}$; see La Vecchia et al. (2020, p.14). 


\section{Simulation Results}

In this section we report the results of a set of Monte Carlo experiments comparing the finite sample performance of different methods for the construction of confidence intervals and regions for the parameters of the mean regression model when there is heteroskedasticity and autocorrelation in the data. We investigate confidence intervals and regions employing $\mathrm{KBB}, \mathrm{MBB}$ and $\mathrm{TBB}$ critical values as well as those based on standard normal or chi-square critical values.

\subsection{Design}

We consider the same simulation design as that of Andrews (1991, Section 9, pp.840-849) and Andrews and Monahan (1992, Section 3, pp.956-964), i.e., linear regression with an intercept and four regressor variables. The model studied is

$$
y_{t}=\beta_{0}+\beta_{1} x_{1, t}+\beta_{2} x_{2, t}+\beta_{3} x_{3, t}+\beta_{4} x_{4, t}+\sigma_{t} u_{t}
$$

where $\sigma_{t}$ is a function of the regressors $x_{i, t},(i=1, \ldots, 4)$, to be specified below. The interest concerns $95 \%$ confidence interval estimators for the coefficient $\beta_{1}$ of the first non-constant regressor.

The regressors and error term $u_{t}$ are generated as follows. First,

$$
u_{t}=\rho u_{t-1}+\varepsilon_{0, t}
$$

with initial condition $u_{-49}=\varepsilon_{0,-49}$. Let

$$
\tilde{x}_{i, t}=\rho \tilde{x}_{i, t-1}+\varepsilon_{i, t},(i=1, \ldots, 4),
$$

with initial conditions $\tilde{x}_{i,-49}=\varepsilon_{i,-49},(i=1, \ldots, 4)$. As in Andrews (1991), the innovations $\varepsilon_{i t},(i=0, \ldots, 4),(t=-49, \ldots, T)$, are independent standard normal random variates. Define $\tilde{x}_{t}=\left(\tilde{x}_{1, t}, \ldots, \tilde{x}_{4, t}\right)^{\prime}$ and $\bar{x}_{t}=\tilde{x}_{t}-\sum_{s=1}^{T} \tilde{x}_{s} / T$. The regressors $x_{i, t},(i=1, \ldots, 4)$, are then constructed as in

$$
\begin{aligned}
x_{t} & =\left(x_{1, t}, \ldots, x_{4, t}\right)^{\prime} \\
& =\left[\sum_{s=1}^{T} \bar{x}_{s} \bar{x}_{s}^{\prime} / T\right]^{-1 / 2} \bar{x}_{t},(t=1, \ldots, T) .
\end{aligned}
$$

The observations on the dependent variable $y_{t}$ are obtained from the linear regression model (4.1) with the true parameter values by invariance set as $\beta_{i}=0,(i=0, \ldots, 4)$, without loss of generality. 
The values of $\rho$ are 0.0, 0.2, 0.5, 0.7 and 0.9. Homoskedastic, $\sigma_{t}=1$, and heteroskedastic, $\sigma_{t}=\left|x_{1 t}\right|$, regression errors are examined. Sample sizes $T=64,128$ and 256 are considered.

The number of bootstrap replications for each experiment was 1000 with 5000 random samples generated.

\subsection{Bootstrap Methods}

Confidence intervals based on KBB are compared with those obtained for MBB [Fitzenberger (1997), Gonçalves and White (2004)] and TBB [Paparoditis and Politis (2002)] for least squares (LS) estimation of (4.1). For succinctness, only the results on the standard percentile bootstrap confidence intervals, Efron (1979), are presented. ${ }^{1}$

To describe the standard percentile KBB method, let $\hat{\beta}_{1}$ denote the LS estimator of $\beta_{1}$ and $\hat{\beta}_{1}^{*}$ its bootstrap counterpart. Because the asymptotic distribution of the LS estimator $\hat{\beta}_{1}$ is normal and hence symmetric about $\beta_{1}$, in large samples the distributions of $\hat{\beta}_{1}-\beta_{1}$ and $\beta_{1}-\hat{\beta}_{1}$ are the same. From the uniform consistency of the bootstrap, Theorem 3.2, the distribution of $\beta_{1}-\hat{\beta}_{1}$ is well approximated by the distribution of $\hat{\beta}_{1}^{*}-\hat{\beta}_{1}$. Therefore, the bootstrap percentile confidence interval for $\beta_{1}$ is given by

$$
\left(\left[1-\frac{1}{k^{1 / 2}}\right] \hat{\beta}_{1}+\frac{\hat{\beta}_{1,0.025}^{*}}{k^{1 / 2}},\left[1-\frac{1}{k^{1 / 2}}\right] \hat{\beta}_{1}+\frac{\hat{\beta}_{1,0.975}^{*}}{k^{1 / 2}}\right),
$$

where $\hat{\beta}_{1, \alpha}^{*}$ is the $100 \alpha$ percentile of the distribution of $\hat{\beta}_{1}^{*}$ and, recall, $k=k_{2} / k_{1}^{2}{ }^{2}$ For $\mathrm{MBB}, k=1$.

KBB confidence intervals are constructed with the following choices of kernel function $k(\cdot)$ : truncated, Bartlett and (2.4) kernel functions, which respectively induce the Bartlett [BT], Smith (2011, Example 2.1, p.1203), Parzen [Pz], Smith (2011, Example 2.2, pp.12031204), and the optimal quadratic spectral [QS] (2.5), Smith (2011, Example 2.3, p.1204), kernel functions $k^{*}(\cdot)$ as the associated convolutions, and the kernel function [PP] based on the optimal trapezoidal taper of Paparoditis and Politis (2001), see Paparoditis and Politis (2001, p.1111). The respective confidence interval estimators are denoted by $\mathrm{KBB}_{\mathrm{J}}$, where $\mathrm{J}=\mathrm{BT}, \mathrm{PZ}, \mathrm{QS}$ and PP. Percentile bootstrap confidence intervals based

\footnotetext{
${ }^{1}$ The standard percentile method is valid here because the asymptotic distribution of the LS estimator is symmetric; see Politis (1998, p.45). Empirical rejection rates for bootstrap confidence intervals based on the symmetric percentile and the equal-tailed methods, Hall $(1992$, p.12), were also computed and are available upon request.

${ }^{2}$ Bootstrap intervals based on $\hat{k}$ were also computed with results similar to those obtained with $k$; see Remark 3.3.
} 
on Corollary 3.1 are denoted $\mathrm{KBB}_{\mathrm{J}}^{a}$, while those based on Remarks 3.4 and 3.5 are denoted by $\mathrm{KBB}_{\mathrm{J}}^{b}$ and $\mathrm{KBB}_{\mathrm{J}}^{c}$ respectively. ${ }^{3,4} \mathrm{~A}$ similar notation is adopted for bootstrap confidence intervals based on MBB and TBB where the latter is computed using the optimal Paparoditis and Politis (2001) trapezoidal taper. The validity of the MBB confidence intervals follows from results to be found in Fitzenberger (1997) and Gonçalves and White (2004). Although Paparoditis and Politis (2002) only provides a theoretical justification for $\mathrm{TBB}^{b}$, the validity of the other TBB confidence intervals follows using versions of results in this paper adapted for TBB.

Standard $t$-statistic confidence intervals using heteroskedastic autocorrelation consistent (HAC) estimators for the asymptotic variance matrix $B_{0}$ are considered based on truncated [TR], Bartlett [BT], Parzen [PZ], Tukey-Hanning [TH] and quadratic spectral [QS] kernel functions $k^{*}(\cdot)$; see Andrews (1991). Alternative $t$-statistic confidence intervals based on the Smith (2005) HAC estimator of $B_{0}$, cf. Remark 3.7, are also examined which use kernel functions $k(\cdot)$ that induce Bartlett $\left[\mathrm{S}_{\mathrm{BT}}\right]$, Parzen $\left[\mathrm{S}_{\mathrm{Pz}}\right]$ and quadratic spectral $\left[\mathrm{S}_{\mathrm{QS}}\right]$ kernels $k^{*}(\cdot)$ respectively and the optimal Paparoditis and Politis (2001) trapezoidal taper $\left[\mathrm{S}_{\mathrm{PP}}\right] \cdot{ }^{5}$

Confidence regions for the full vector of regression parameters $\left(\beta_{0}, \beta_{1}, \beta_{2}, \beta_{3}, \beta_{4}\right)$ based on the standard normal distribution-based LR statistic $[\mathcal{L R}]$ and standard HAC Wald statistic $[\mathcal{W}]$ are examined. A conservative confidence interval estimator for $\beta_{1}$ may then be constructed using the "projection" method [Dufour (2003, Section 6.2, 791-792)] with nominal coverage probability that of the full vector confidence region. The statistic $\mathcal{L} \mathcal{R}$ is computed as sample size $T$ multiplied by the difference between the sum of squared unrestricted LS residuals and the sum of the squares of the dependent variable whereas the standard Wald statistic $\mathcal{W}$ uses the estimators $-\sum_{t=1}^{T} x_{t} x_{t}^{\prime} / T$ for $A_{0}=-\mathrm{E}\left[x_{t} x_{t}^{\prime}\right]$ and kernel-based HAC variance estimators for $B_{0}=\operatorname{var}\left[T^{-1 / 2} \sum_{t=1}^{T} x_{t} \sigma_{t} u_{t}\right]$ as enumerated above, where $x_{t}=\left(1, x_{1, t}, x_{2, t}, x_{3, t}, x_{4, t}\right)^{\prime}$. The relevant bootstrap critical values for $\mathcal{L} \mathcal{R}$ based confidence regions are computed based on Theorem 3.3 whereas, since $\mathcal{W}$ converges in distribution to $\chi_{5}^{2}(\cdot)$, prob- $\mathcal{P}$, those based on the standard $\mathcal{W}$-statistic use the nominal $\chi_{5}^{2}(\cdot)$ critical values. The statistic $\mathcal{L} \mathcal{R}$ converges in distribution to $\mathcal{M}_{5}(\cdot, \lambda)$, prob- $\mathcal{P}$, a

\footnotetext{
${ }^{3}$ Uncentred bootstrap confidence intervals, cf. Remark 3.6, were also computed with results similar to the respective centred versions from Corollary 3.1 and Remarks 3.4 and 3.5.

${ }^{4}$ Since $\hat{\theta}^{*}-\hat{\theta}=-\left[\partial^{2} \overline{\mathcal{L}}_{m_{T}}^{*}(\hat{\theta}) / \partial \theta \partial \theta^{\prime}\right]^{-1} \partial \overline{\mathcal{L}}_{m_{T}}^{*}(\hat{\theta}) / \partial \theta$ for the LS estimator, bootstrap confidence intervals based on Theorem 3.2 are numerically identical to those based on the uncentred Corollary 3.1 bootstrap.

${ }^{5}$ The HAC estimator of $B_{0}$ of Andrews (1991) is given by $\sum_{s=1-T}^{T-1} k^{*}\left(s / S_{T}\right) \hat{R}_{T}(s)$ where the sample autocovariance $\hat{R}_{T}(s)=T^{-1} \sum_{t=\max [1,1-s]}^{\min [T, T}\left(\partial \mathcal{L}_{t}(\hat{\theta}) / \partial \theta\right)\left(\partial \mathcal{L}_{t-s}(\hat{\theta}) / \partial \theta\right)^{\prime},(s=1-T, \ldots, T-1)$, while the Smith (2005) HAC estimator of $B_{0}$ is $T^{-1} \sum_{t=1}^{T}\left(\partial \mathcal{L}_{t T}(\hat{\theta}) / \partial \theta\right)\left(\partial \mathcal{L}_{t T}(\hat{\theta}) / \partial \theta\right)^{\prime}$, cf. (2.2).
} 
weighted sum of 5 independent chi-square distributed random variables each with one degree of freedom and each weighted by successive elements of $\lambda$ the 5 -dimensional vector of eigenvalues of $-B_{0} A_{0}^{-1}$. The $\mathcal{M}_{5}(\cdot, \lambda)$ distribution is approximated via simulation using 5000 replications replacing $A_{0}$ and $B_{0}$ as described above. The same notation as that above is employed for the HAC kernel function choices.

\subsection{Bandwidth Choice}

The accuracy of the bootstrap approximation in practice is particularly sensitive to the choice of the bandwidth or block size $S_{T}$. Gonçalves and White (2004) suggests basing the choice of MBB block size on the optimal automatic bandwidth, see Andrews (1991, Section 5, pp.830-832), appropriate for HAC variance matrix estimation using the Bartlett kernel, noting that the MBB bootstrap variance estimator is asymptotically equivalent to the Bartlett kernel variance estimator. Smith (2011, Lemma A.3, p.1219) obtained a similar equivalence between the KBB variance estimator and the corresponding HAC estimator based on the induced kernel function $k^{*}(\cdot)$; see also Smith (2005, Lemma 2.1, p.164). We adopt a similar approach to that of Gonçalves and White (2004) to the choice of the bandwidth for KBB confidence interval estimators. However, rather than using the method suggested in Andrews (1991) for estimation of the optimal automatic bandwidth for the induced kernel function $k^{*}(\cdot)$, a non-parametric estimator of this bandwidth is adopted; see Politis and Romano (1995). Despite lacking a theoretical justification, the results discussed below indicate that this procedure fares well for the simulation designs studied here. ${ }^{6}$

The infeasible optimal bandwidth for HAC variance matrix estimation based on the kernel $k^{*}(\cdot)$ is given by

$$
S_{T}^{*}=\left(q\left(k_{(q)}^{*}\right)^{2} \alpha(q) T / \int_{-\infty}^{\infty} k^{*}(x)^{2} d x\right)^{1 /(2 q+1)},
$$

where $k_{(q)}^{*}=\lim _{y \rightarrow 0}\left[1-k^{*}(y)\right] /|y|^{q}$ and $\alpha(q)=2 \operatorname{vec}\left(\sum_{s=-\infty}^{\infty}|s|^{2} R(s)\right)^{\prime} W \operatorname{vec}\left(\sum_{s=-\infty}^{\infty}|s|^{2} R(s)\right)$ $/ \operatorname{tr}\left(W\left(I_{p p}+K_{p p}\right)\left(\Sigma_{\infty} \otimes \Sigma_{\infty}\right)\right), q \in[0, \infty)$, cf. the optimal KBB bandwidth $S_{T}^{*}$ of section 2 when $q=2$; see Andrews (1991, (5.2), p.830). Note that $q=1$ for the Bartlett [BT]

\footnotetext{
${ }^{6}$ The bandwidth choice is estimation-optimal minimising the asymptotic mean squared error of the corresponding $\mathrm{HAC}$ variance estimator. In general, the bandwidth choice here should be test-optimal and would differ from that pertinent for estimation. Using Edgeworth expansions with joint sample size $T \rightarrow \infty$ and bandwidth $S_{T}=b T$ with $b \rightarrow 0$ asymptotics, Sun et al. (2008) develop an optimal bandwidth choice for a nonparametrically studentised $t$-test of a two-sided hypothesis in the location model with heteroskedastic and dependent innovations that minimises a weighted average of Type I and Type II errors. See, in particular, Sun et al. (2008), Steps 1-9, 177-178).
} 
kernel and $q=2$ for the Parzen [Pz], quadratic spectral [QS] kernels and the optimal Paparoditis and Politis (2001) taper [PP]. In the linear regression model (4.1) context, with diagonal weight matrix $W=\operatorname{diag}\left(w_{1}, \ldots, w_{4}\right)$,

$$
\alpha(q)=\frac{\sum_{i=1}^{4} w_{i}\left[\sum_{s=-\infty}^{\infty}|s|^{q} R_{i}(s)\right]^{2}}{\sum_{i=1}^{4} w_{i}\left[\sum_{s=-\infty}^{\infty} R_{i}(s)\right]^{2}},
$$

where $R_{i}(s)$ is the $s$ th autocovariance of $x_{i t}\left(y_{t}-\sum_{k=1}^{4} x_{k t} \beta_{k}\right),(i=1, \ldots, 4)$. As in the Monte Carlo study Andrews (1991, section 9, pp.840-849), unit weights $w_{i}=1$, $(i=1, \ldots, 4)$, are chosen.

The optimal bandwidth $S_{T}^{*}$ requires the estimation of the parameters $\alpha(1)$ and $\alpha(2)$. Rather then base estimation of $\alpha(q)$ on a particular ARMA model as suggested in Andrews (1991, Section 6, pp.832-837), a feasible non-parametric estimator of the Andrews (1991) optimal bandwidth replaces $\alpha(q)$ by a consistent estimator based on the flat-top lag-window of Politis and Romano (1995), viz.

$$
\hat{\alpha}(q)=\frac{\sum_{i=1}^{4}\left[\sum_{j=-M_{i}}^{M_{i}}|j|^{q} \lambda\left(\frac{j}{M_{i}}\right) \hat{R}_{i}(j)\right]^{2}}{\sum_{i=1}^{4}\left[\sum_{j=-M_{i}}^{M_{i}} \lambda\left(\frac{j}{M_{i}}\right) \hat{R}_{i}(j)\right]^{2}},(q=1,2),
$$

where $\lambda(t)=\mathbb{I}(|t| \in[0,1 / 2])+2(1-|t|) \mathbb{I}(|t| \in(1 / 2,1]), \hat{R}_{i}(j)$ is the sample $j$ th autocovariance of $\left\{x_{i t}\left(y_{t}-\sum_{k=1}^{4} x_{k t} \beta_{k}\right)\right\},(i=1, \ldots, 4)$, using LS estimation of $\beta_{k}, \quad(k=$ $1, \ldots, 4)$, and $M_{i},(i=1, \ldots, 4)$, are computed using the method described in Politis and White (2004, ftn. c, p.59). The feasible optimal bandwidth estimator is then $\hat{S}_{T}^{*}=\left(q\left(k_{(q)}^{*}\right)^{2} \hat{\alpha}(q) T / \int_{-\infty}^{\infty} k^{*}(x)^{2} d x\right)^{1 /(2 q+1)}$ whereas the bandwidth formula $S_{T}^{*}=$ $0.6611(\hat{\alpha}(2) T)^{1 / 5}$ is used for the truncated kernel [TR] HAC estimator, see Andrews (1991, ftn.5, p. 834).

Bootstrap sample sizes are defined as $m_{T}=\max \left[\left\lfloor T / \hat{S}_{T}^{*}\right\rfloor, 1\right]$, where $\lfloor\cdot\rfloor$ is the floor function. $\mathrm{MBB}$ and $\mathrm{TBB}$ block sizes are given by $\min \left[\left\lceil\hat{S}_{T}^{*}\right\rceil, T\right]$, where $\lceil\cdot\urcorner$ is the ceiling function and $S_{T}^{*}$ the optimal bandwidth estimator for the Bartlett kernel $k^{*}(\cdot)$ for MBB and for the kernel $k^{*}(\cdot)$ induced by the optimal Paparoditis and Politis (2001) trapezoidal taper for TBB.

\subsection{Results}

\subsubsection{Confidence Intervals}

Tables 1 and 2 provide the empirical coverage rates for $95 \%$ confidence interval estimates obtained using the methods described above for the homoskedastic and heteroskedastic 
cases respectively.

\section{Tables 1 and 2 around here}

Overall, to a lesser or greater degree, all confidence interval estimates display undercoverage for the true value $\beta_{1}=0$ but especially for high values of $\rho$, a feature found in previous studies of MBB, see, e.g., Gonçalves and White (2004), and confidence intervals based on $t$-statistics with HAC variance matrix estimators, see Andrews (1991). As should be expected from the theoretical results of Section 3, as $T$ increases, empirical coverage rates approach the nominal rate of $95 \%$.

Additionally, Tables 1 and 2 reveal that the empirical coverage rates of the bootstrap confidence intervals based on Corollary 3.1 are very similar to those based on Theorem 3.2, although the former corresponds to a centred version of the latter, see ftn. 4, and is intuitively expected to yield improvements, cf. Paparoditis and Politis (2001, p.1108). Furthermore, the empirical coverage rates of the bootstrap confidence intervals constructed using the results in Remarks 3.4 and 3.5 are systematically lower across KBB, MBB and TBB than those based on Theorem 3.2 and Corollary 3.1. With a few exceptions, all bootstrap confidence interval estimates outperform those based on HAC $t$-statistics for all values of $\rho$ and for all sample sizes except for $T=256$ when, for lower and moderate values, both bootstrap and HAC $t$-statistic methods produce similarly satisfactory results. The following discussion is therefore conducted based solely on KBB, MBB and TBB.

A comparison of the various KBB confidence interval estimates for the homoskedastic design in Table 1 for $T=64$ with those using MBB reveals that generally, for low values of $\rho$, the coverage rates for $\mathrm{KBB}_{\mathrm{Bт}}$ are closer to the nominal $95 \%$ than those of $\mathrm{MBB}$, although both are based on the truncated kernel, and other KBB methods. Furthermore, $\mathrm{KBB}_{\mathrm{PP}}$ is superior to $\mathrm{KBB}_{\mathrm{BT}}, \mathrm{KBB}_{\mathrm{PZ}}$ and $\mathrm{KBB}_{\mathrm{QS}}$ for high values of $\rho$, although not dramatically so for moderate $\rho$. While both bootstraps use the same kernel function, MBB has similar coverage rates to $\mathrm{KBB}_{\mathrm{BT}}$ for low to moderate $\rho$ but higher coverage rates for the higher values of $\rho$. TBB coverage is poorer than MBB at low values of $\rho$ and is dominated by $\mathrm{KBB}_{\mathrm{PP}}$ at all values of $\rho$ even though both methods use the same taper/kernel. A similar pattern is repeated for the larger sample size $T=128$ although the differences across bootstrap methods narrow. For sample size $T=256$, all bootstrap and HAC $t$-statistic confidence intervals display similar coverage rates except for $\rho=0.9$ when $\mathrm{KBB}_{\mathrm{PP}}$ is superior. Overall, the results with homoskedastic innovations in Table 1 indicate that $\mathrm{KBB}_{\mathrm{PP}}$ is the superior bootstrap method at moderate to high values of $\rho$ at all sample sizes with $\mathrm{KBB}_{\mathrm{BT}}, \mathrm{KBB}_{\mathrm{QS}}, \mathrm{KBB}_{\mathrm{PP}}$ and $\mathrm{TBB}$ reasonably competitive for 
the lower $\rho$ at the larger sample sizes.

In Table 2, for heteroskedastic innovations, the differences in coverage rates between the various methods narrow and are more varied. For sample size $T=64$, all $\mathrm{KBB}$ bootstrap confidence intervals display similar coverage for low $\rho$ but $\mathrm{KBB}_{\mathrm{QS}}$ and $\mathrm{KBB}_{\mathrm{PP}}$ are superior and perform similarly for moderate to high values of $\rho$ and for all sample sizes. $\mathrm{MBB}$ is again dominated by $\mathrm{KBB}_{\mathrm{BT}}$ and, likewise, $\mathrm{KBB}_{\mathrm{PP}}$ is superior to $\mathrm{TBB}$ at all sample sizes.

\subsubsection{Confidence Regions}

Empirical coverage rates for $95 \%$ confidence region estimates based on $\mathcal{L} \mathcal{R}$ and $\mathcal{W}$ statistics with homoskedastic and heteroskedastic innovations are presented in Tables 3 and 4 respectively.

\section{Tables 3 and 4 around here}

Overall, all methods, using either bootstrap or nominal critical values, display sometimes substantial undercoverage in small samples, although the $\mathcal{L} \mathcal{R}$-based confidence regions tend to display empirical coverage rates closer to the nominal $95 \%$ level than those based on the standard $\mathcal{W}$ statistic. The performance of all methods seriously deteriorates as $\rho$ increases but improves as sample size $T$ increases.

Among the $\mathcal{L} \mathcal{R}$-based confidence regions, those based on $\mathrm{KBB}_{\mathrm{PZ}}$ and Tukey-Hanning $[\mathrm{TH}]$ kernel nominal critical values achieve the best performance. In general, empirical coverage rates based on bootstrap and on first order asymptotic theory are broadly similar. Of the $\mathcal{W}$-based confidence regions, those based on the truncated [TR] kernel display the superior performance when dependence is weak $[\rho=0.2]$ but those using the $\mathrm{TH}$ kernel behave better with stronger dependence. In general, however, the $\mathcal{W}$-based confidence regions display substantial undercoverage. Surprisingly, perhaps, the behaviour of both $\mathcal{L} \mathcal{R}$ - and $\mathcal{W}$-based confidence regions improves under the heteroskedastic relative to the homoskedastic design.

\subsection{SUMmary}

In general, for homoskedastic innovations, confidence interval estimates based on $\mathrm{KBB}_{\mathrm{PP}}$ provide the best coverage rates for all values of $\rho$ and sample sizes whereas, under heteroskedasticity, the performance of $\mathrm{KBB}_{\mathrm{BT}}, \mathrm{KBB}_{\mathrm{QS}}$ and $\mathrm{KBB}_{\mathrm{PP}}$ confidence intervals are similar and dominate for low and moderate values of $\rho$ and the larger sample sizes. 
$\mathrm{KBB}_{\mathrm{QS}}$ is broadly competitive at all values of $\rho$ except at $\rho=0.9$ for homoskedastic innovations.

Overall, confidence region estimators for the full vector of parameters cannot be recommended with any confidence.

\section{CONCLUSiON}

This paper applies the kernel block bootstrap method to quasi-maximum likelihood estimation of dynamic models under stationarity and weak dependence. The proposed bootstrap method is simple to implement by first kernel-weighting the components comprising the quasi-log likelihood function appropriately and then sampling the resultant transformed components using the standard " $m$ out of $n$ " bootstrap.

We investigate the first order asymptotic properties of the kernel block bootstrap for quasi-maximum likelihood demonstrating, in particular, its consistency and the firstorder asymptotic validity of the bootstrap approximation to the distribution of the quasimaximum likelihood estimator. A number of first order equivalent kernel block bootstrap schemes are suggested of differing computational complexities. A set of simulation experiments for the mean linear regression model illustrates the efficacy of the kernel block bootstrap for quasi-maximum likelihood estimation. Indeed, in these experiments, the kernel block bootstrap outperforms other bootstrap methods for the sample sizes considered, especially if the induced KBB kernel function is chosen appropriately as either the Bartlett kernel or the quadratic spectral kernel or the optimal taper of Paparoditis and Politis (2001) is used to kernel-weight the quasi-log likelihood function.

\section{APPENDIX}

Throughout the Appendices, $C$ and $\Delta$ denote generic positive constants that may be different in different uses with $\mathrm{C}, \mathrm{M}$, and $\mathrm{T}$ the Chebyshev, Markov, and triangle inequalities respectively. UWL is a uniform weak law of large numbers such as Newey and McFadden (1994, Lemma 2.4, p.2129) for stationary and mixing (and, thus, ergodic) processes.

A similar notation is adopted to that in Gonçalves and White (2004). For any bootstrap statistic $T^{*}(\cdot, \omega), T^{*}(\cdot, \omega) \rightarrow 0$, prob- $\mathcal{P}_{\omega}^{*}$, prob- $\mathcal{P}$ if, for any $\delta>0$ and any $\xi>0$, $\lim _{T \rightarrow \infty} \mathcal{P}\left\{\omega: \mathcal{P}_{\omega}^{*}\left\{\lambda:\left|T^{*}(\lambda, \omega)\right|>\delta\right\}>\xi\right\}=0$.

To simplify the analysis, the appendices consider the transformed uncentred observa- 
tions

$$
\mathcal{L}_{t T}(\theta)=\frac{1}{\left(k_{2} S_{T}\right)^{1 / 2}} \sum_{s=t-1}^{t-T} k\left(\frac{s}{S_{T}}\right) \mathcal{L}_{t-s}(\theta)
$$

with $k_{2}$ substituting for $\hat{k}_{2}=S_{T}^{-1} \sum_{t=1-T}^{T-1} k\left(t / S_{T}\right)^{2}$ in the main text since $\hat{k}_{2}-k_{2}=o(1)$, cf. PS Supplement Corollary K.2, p.S.21.

For simplicity, where required, it is assumed $T / S_{T}$ is integer.

\section{Appendix A: Preliminary Lemmas}

Assumption A.1. (Bootstrap Pointwise WLLN.) For each $\theta \in \Theta \subset \mathcal{R}^{d_{\theta}}, \Theta$ a compact set, $S_{T} \rightarrow \infty$ and $S_{T}=o\left(T^{-1 / 2}\right)$

$$
\left(k_{2} / S_{T}\right)^{1 / 2}\left[\overline{\mathcal{L}}_{m_{T}}^{*}(\theta)-\overline{\mathcal{L}}_{T}(\theta)\right] \rightarrow 0, \text { prob- } \mathcal{P}_{\omega}^{*}, \text { prob- } \mathcal{P} .
$$

Remark A.1. See Lemma A.2 below.

Assumption A.2. (Uniform Convergence.)

$$
\sup _{\theta \in \Theta}\left|\left(k_{2} / S_{T}\right)^{1 / 2} \overline{\mathcal{L}}_{T}(\theta)-k_{1} \overline{\mathcal{L}}(\theta)\right| \rightarrow 0 \text { prob-P } .
$$

Remark A.2. The hypotheses of the UWLs Smith (2011, Lemma A.1, p.1217) and Newey and McFadden (1994, Lemma 2.4, p.2129) for stationary and mixing (and, thus, ergodic) processes are satisfied under Assumptions 3.1-3.3. Hence, noting $\left.\sup _{\theta \in \Theta} \| \overline{\mathcal{L}}(\theta)-\mathcal{L}(\theta)\right] \|$ $\rightarrow 0$, prob- $\mathcal{P}$, where $\left.\mathcal{L}(\theta)=\mathrm{E}\left[\mathcal{L}_{t}(\theta)\right], \sup _{\theta \in \Theta} \|\left(k_{2} / S_{T}\right)^{1 / 2} \overline{\mathcal{L}}_{T}(\theta)-k_{1} \mathcal{L}(\theta)\right] \| \rightarrow 0$, prob- $\mathcal{P}$. Thus, Assumption A.2 follows by $\mathrm{T}$ and $k_{1}, k_{2}=O(1)$.

Assumption A.3. (Global Lipschitz Continuity.) For all $\theta, \theta^{0} \in \Theta,\left|\mathcal{L}_{t}(\theta)-\mathcal{L}_{t}\left(\theta^{0}\right)\right| \leq$ $L_{t}\left\|\theta-\theta^{0}\right\|$ a.s. $\mathcal{P}$ where $\sup _{T} E\left[\sum_{t=1}^{T} L_{t} / T\right]<\infty$.

Remark A.3. Assumption A.3 is Assumption 3.2(c).

Lemma A.1. (Bootstrap UWL.) Suppose Assumptions A.1-A.3 hold. Then, for $S_{T} \rightarrow \infty$ and $S_{T}=o\left(T^{1 / 2}\right)$,

$$
\sup _{\theta \in \Theta}\left|\left(k_{2} / S_{T}\right)^{1 / 2} \overline{\mathcal{L}}_{m_{T}}^{*}(\theta)-k_{1} \overline{\mathcal{L}}(\theta)\right| \rightarrow 0, \text { prob- } \mathcal{P}_{\omega}^{*}, \text { prob-} \mathcal{P} .
$$

Proof. From Assumption A.2 the result is proven if

$$
\sup _{\theta \in \Theta}\left(k_{2} / S_{T}\right)^{1 / 2}\left|\overline{\mathcal{L}}_{m_{T}}^{*}(\theta)-\overline{\mathcal{L}}_{T}(\theta)\right| \rightarrow 0, \text { prob- } \mathcal{P}_{\omega}^{*}, \text { prob- } \mathcal{P} .
$$


The following preliminary results are useful in the later analysis. By global Lipschitz continuity of $\mathcal{L}_{t}(\cdot)$ and by $\mathrm{T}$, for $T$ large enough,

$$
\begin{aligned}
\left.\left(k_{2} / S_{T}\right)^{1 / 2} \mid \overline{\mathcal{L}}_{T}(\theta)\right)-\overline{\mathcal{L}}_{T}\left(\theta^{0}\right) \mid & \leq \frac{1}{T} \sum_{t=1}^{T} \frac{1}{S_{T}} \sum_{s=t-T}^{t-1}\left|k\left(\frac{s}{S_{T}}\right)\right|\left|\mathcal{L}_{t-s}(\theta)-\mathcal{L}_{t-s}\left(\theta^{0}\right)\right| \\
& =\frac{1}{T} \sum_{t=1}^{T}\left|\mathcal{L}_{t}(\theta)-\mathcal{L}_{t}\left(\theta^{0}\right)\right|\left|\frac{1}{S_{T}} \sum_{s=1-t}^{T-t} k\left(\frac{s}{S_{T}}\right)\right| \\
& \leq C\left\|\theta-\theta^{0}\right\| \frac{1}{T} \sum_{t=1}^{T} L_{t}
\end{aligned}
$$

since for some $0<C<\infty$

$$
\left|\frac{1}{S_{T}} \sum_{s=1-t}^{T-t} k\left(\frac{s}{S_{T}}\right)\right| \leq O(1)<C
$$

uniformly $t$ for $T$ large enough, see Smith (2011, eq. (A.5), p.1218). Next, for some $0<C^{*}<\infty$,

$$
\begin{aligned}
\left(k_{2} / S_{T}\right)^{1 / 2} E^{*}\left[\left|\overline{\mathcal{L}}_{m_{T}}^{*}(\theta)-\overline{\mathcal{L}}_{m_{T}}^{*}\left(\theta^{0}\right)\right|\right] & =\frac{1}{m_{T}} \sum_{s=1}^{m_{T}} \frac{1}{S_{T}} E^{*}\left[\sum_{r=t_{s}^{*}-T}^{t_{s}^{*}-1}\left|k\left(\frac{r}{S_{T}}\right)\right|\left|\mathcal{L}_{t_{s}^{*}-r}(\theta)-\mathcal{L}_{t_{s}^{*}-r}\left(\theta^{0}\right)\right|\right] \\
& =\frac{1}{T} \sum_{t=1}^{T}\left|\mathcal{L}_{t}(\theta)-\mathcal{L}_{t}\left(\theta^{0}\right)\right| \frac{1}{S_{T}} \sum_{r=t-T}^{t-1}\left|k\left(\frac{r}{S_{T}}\right)\right| \\
& \leq C^{*}\left\|\theta-\theta^{0}\right\| \frac{1}{T} \sum_{t=1}^{T} L_{t} .
\end{aligned}
$$

Hence, by $\mathrm{M}$, for some $0<C^{*}<\infty$ uniformly $t$ for large enough $T$,

$$
\mathcal{P}_{\omega}^{*}\left\{\left(k_{2} / S_{T}\right)^{1 / 2}\left|\overline{\mathcal{L}}_{m_{T}}^{*}(\theta)-\overline{\mathcal{L}}_{m_{T}}^{*}\left(\theta^{0}\right)\right|>\epsilon\right\} \leq \frac{C^{*}}{\epsilon}\left\|\theta-\theta^{0}\right\| \frac{1}{T} \sum_{t=1}^{T} L_{t} .
$$

The remaining part of the proof is identical to Gonçalves and White (2000, Proof of Lemma A.2, pp.30-31) and is given here for completeness; cf. Hall and Horowitz (1996, Proof of Lemma 8, p.913). Given $\varepsilon>0$, let $\left\{\eta\left(\theta_{i}, \varepsilon\right),(i=1, \ldots, I)\right\}$ denote a finite subcover of $\Theta$ where $\eta\left(\theta_{i}, \varepsilon\right)=\left\{\theta \in \Theta:\left\|\theta-\theta_{i}\right\|<\varepsilon\right\},(i=1, \ldots, I)$. Now

$$
\sup _{\theta \in \Theta}\left(k_{2} / S_{T}\right)^{1 / 2}\left|\overline{\mathcal{L}}_{m_{T}}^{*}(\theta)-\overline{\mathcal{L}}_{T}(\theta)\right|=\max _{i=1, \ldots, I} \sup _{\theta \in \eta\left(\theta_{i}, \varepsilon\right)}\left(k_{2} / S_{T}\right)^{1 / 2}\left|\overline{\mathcal{L}}_{m_{T}}^{*}(\theta)-\overline{\mathcal{L}}_{T}(\theta)\right| .
$$

The argument $\omega \in \Omega$ is omitted for brevity as in Gonçalves and White (2000). It then follows that, for any $\delta>0$ (and any fixed $\omega$ ),

$\mathcal{P}_{\omega}^{*}\left\{\sup _{\theta \in \Theta}\left(k_{2} / S_{T}\right)^{1 / 2}\left|\overline{\mathcal{L}}_{m_{T}}^{*}(\theta)-\overline{\mathcal{L}}_{T}(\theta)\right|>\delta\right\} \leq \sum_{i=1}^{I} \mathcal{P}_{\omega}^{*}\left\{\sup _{\theta \in \eta\left(\theta_{i}, \varepsilon\right)}\left(k_{2} / S_{T}\right)^{1 / 2}\left|\overline{\mathcal{L}}_{m_{T}}^{*}(\theta)-\overline{\mathcal{L}}_{T}(\theta)\right|>\delta\right\}$. 
For any $\theta \in \eta\left(\theta_{i}, \varepsilon\right)$, by $\mathrm{T}$,

$$
\begin{aligned}
\left(k_{2} / S_{T}\right)^{1 / 2}\left|\overline{\mathcal{L}}_{m_{T}}^{*}(\theta)-\overline{\mathcal{L}}_{T}(\theta)\right| \leq & \left(k_{2} / S_{T}\right)^{1 / 2}\left|\overline{\mathcal{L}}_{m_{T}}^{*}\left(\theta_{i}\right)-\overline{\mathcal{L}}_{T}\left(\theta_{i}\right)\right|+\left(k_{2} / S_{T}\right)^{1 / 2}\left|\overline{\mathcal{L}}_{m_{T}}^{*}(\theta)-\overline{\mathcal{L}}_{m_{T}}^{*}\left(\theta_{i}\right)\right| \\
& +\left(k_{2} / S_{T}\right)^{1 / 2}\left|\overline{\mathcal{L}}_{T}(\theta)-\overline{\mathcal{L}}_{T}\left(\theta_{i}\right)\right|
\end{aligned}
$$

Hence, for any $\delta>0$ and $\xi>0$,

$$
\begin{gathered}
\mathcal{P}\left\{\mathcal{P}_{\omega}^{*}\left\{\sup _{\theta \in \eta\left(\theta_{i}, \varepsilon\right)}\left(k_{2} / S_{T}\right)^{1 / 2}\left|\overline{\mathcal{L}}_{m_{T}}^{*}(\theta)-\overline{\mathcal{L}}_{T}(\theta)\right|>\delta\right\}>\xi\right\} \leq \mathcal{P}\left\{\mathcal{P}_{\omega}^{*}\left\{\left(k_{2} / S_{T}\right)^{1 / 2}\left|\overline{\mathcal{L}}_{m_{T}}^{*}\left(\theta_{i}\right)-\overline{\mathcal{L}}_{T}\left(\theta_{i}\right)\right|>\frac{\delta}{3}\right\}>\frac{\xi}{3}\right\} \\
+\mathcal{P}\left\{\mathcal{P}_{\omega}^{*}\left\{\left(k_{2} / S_{T}\right)^{1 / 2}\left|\overline{\mathcal{L}}_{m_{T}}^{*}(\theta)-\overline{\mathcal{L}}_{m_{T}}^{*}\left(\theta_{i}\right)\right|>\frac{\delta}{3}\right\}>\frac{\xi}{3}\right\} \\
+\mathcal{P}\left\{\left(k_{2} / S_{T}\right)^{1 / 2}\left|\overline{\mathcal{L}}_{T}(\theta)-\overline{\mathcal{L}}_{T}\left(\theta_{i}\right)\right|>\frac{\delta}{3}\right\}
\end{gathered}
$$

By Assumption A.1

$$
\mathcal{P}\left\{\mathcal{P}_{\omega}^{*}\left\{\left(k_{2} / S_{T}\right)^{1 / 2}\left|\overline{\mathcal{L}}_{m_{T}}^{*}\left(\theta_{i}\right)-\overline{\mathcal{L}}_{T}\left(\theta_{i}\right)\right|>\frac{\delta}{3}\right\}>\frac{\xi}{3}\right\}<\frac{\xi}{3}
$$

for large enough $T$. Also, by $\mathrm{M}$ (for fixed $\omega$ ) and Assumption A.3, noting $L_{t} \geq 0$, $(t=1, \ldots, T)$, from eq. (A.2),

$$
\begin{aligned}
\mathcal{P}_{\omega}^{*}\left\{\left(k_{2} / S_{T}\right)^{1 / 2}\left|\overline{\mathcal{L}}_{m_{T}}^{*}(\theta)-\overline{\mathcal{L}}_{m_{T}}^{*}\left(\theta_{i}\right)\right|\right. & \left.>\frac{\delta}{3}\right\} \leq \frac{3 C^{*}}{\delta}\left\|\theta-\theta_{i}\right\| \frac{1}{T} \sum_{t=1}^{T} L_{t} \\
& \leq \frac{3 C^{*} \varepsilon}{\delta} \frac{1}{T} \sum_{t=1}^{T} L_{t} .
\end{aligned}
$$

As a consequence, for any $\delta>0$ and $\xi>0$, for $T$ sufficiently large,

$$
\begin{aligned}
\mathcal{P}\left\{\mathcal { P } _ { \omega } ^ { * } \left\{\left(k_{2} / S_{T}\right)^{1 / 2}\left|\overline{\mathcal{L}}_{m_{T}}^{*}(\theta)-\overline{\mathcal{L}}_{m_{T}}^{*}\left(\theta_{i}\right)\right|\right.\right. & \left.\left.>\frac{\delta}{3}\right\}>\frac{\xi}{3}\right\} \leq \mathcal{P}\left\{\frac{3 C^{*} \varepsilon}{\delta} \frac{1}{T} \sum_{t=1}^{T} L_{t}>\frac{\xi}{3}\right\} \\
& =\mathcal{P}\left\{\frac{1}{T} \sum_{t=1}^{T} L_{t}>\frac{\xi \delta}{9 C^{*} \varepsilon}\right\} \\
& \leq \frac{9 C^{*} \varepsilon}{\xi \delta} \mathrm{E}\left[\frac{1}{T} \sum_{t=1}^{T} L_{t}\right] \\
& \leq \frac{9 C^{*} \varepsilon \Delta}{\xi \delta}<\frac{\xi}{3}
\end{aligned}
$$

for the choice $\varepsilon<\xi^{2} \delta / 27 C^{*} \Delta$, where, since, by hypothesis $\mathrm{E}\left[\sum_{t=1}^{T} L_{t} / T\right]=O(1)$, the second and third inequalities follow respectively from $\mathrm{M}$ and $\Delta$ a sufficiently large but finite constant such that $\sup _{T} \mathrm{E}\left[\sum_{t=1}^{T} L_{t} / T\right]<\Delta$. Similarly, from eq. (A.1), for any $\delta>0$ and $\xi>0$, by Assumption A.3, $\mathcal{P}\left\{\left(k_{2} / S_{T}\right)^{1 / 2}\left|\overline{\mathcal{L}}_{T}(\theta)-\overline{\mathcal{L}}_{T}\left(\theta_{i}\right)\right|>\delta / 3\right\} \leq \mathcal{P}\left\{C \varepsilon \sum_{t=1}^{T} L_{t} / T>\right.$ $\delta / 3\} \leq 3 C \varepsilon \Delta / \delta<\xi / 3$ for $T$ sufficiently large for the choice $\varepsilon<\xi \delta / 9 C \Delta$.

Therefore, from eq. (A.3), the conclusion of the Lemma follows if

$$
\varepsilon=\frac{\xi \delta}{9 \Delta} \max \left(\frac{1}{C}, \frac{\xi}{3 C^{*}}\right)
$$


Lemma A.2. (Bootstrap Pointwise WLLN.) Suppose Assumptions 3.1, 3.2(a) and 3.3 are satisfied. Then, if $T^{1 / \alpha} / m_{T} \rightarrow 0$ and $\mathrm{E}\left[\sup _{\theta \in \Theta}\left|\log f_{t}(\theta)\right|^{\alpha}\right]<\infty$ for some $\alpha>v$, for each $\theta \in \Theta \subset \mathcal{R}^{d_{\theta}}, \Theta$ a compact set,

$$
\left(k_{2} / S_{T}\right)^{1 / 2}\left[\overline{\mathcal{L}}_{m_{T}}^{*}(\theta)-\overline{\mathcal{L}}_{T}(\theta)\right] \rightarrow 0, \text { prob- } \mathcal{P}_{\omega}^{*}, \text { prob- } \mathcal{P}
$$

Proof: The argument $\theta$ is suppressed throughout for brevity. First, cf. Gonçalves and White (2004, Proof of Lemma A.5, p.215),

$$
\left(k_{2} / S_{T}\right)^{1 / 2}\left(\overline{\mathcal{L}}_{m_{T}}^{*}-\overline{\mathcal{L}}_{T}\right)=\left(k_{2} / S_{T}\right)^{1 / 2}\left(\overline{\mathcal{L}}_{m_{T}}^{*}-\mathrm{E}^{*}\left[\overline{\mathcal{L}}_{m_{T}}^{*}\right]\right)-\left(k_{2} / S_{T}\right)^{1 / 2}\left(\overline{\mathcal{L}}_{T}-\mathrm{E}^{*}\left[\overline{\mathcal{L}}_{m_{T}}^{*}\right]\right)
$$

Since $\mathrm{E}^{*}\left[\overline{\mathcal{L}}_{m_{T}}^{*}\right]=\overline{\mathcal{L}}_{T}$, cf. PS (Section 2.2, pp.2-3), the second term $\overline{\mathcal{L}}_{T}-\mathrm{E}^{*}\left[\overline{\mathcal{L}}_{m_{T}}^{*}\right]$ is zero. Hence, the result follows if, for any $\delta>0$ and $\xi>0$ and large enough $T$, $\mathcal{P}\left\{\mathcal{P}_{\omega}^{*}\left\{\left(k_{2} / S_{T}\right)^{1 / 2}\left|\overline{\mathcal{L}}_{m_{T}}^{*}-\mathrm{E}^{*}\left[\overline{\mathcal{L}}_{m_{T}}^{*}\right]\right|>\delta\right\}>\xi\right\}<\xi$.

Without loss of generality, set $\mathrm{E}^{*}\left[\overline{\mathcal{L}}_{m_{T}}^{*}\right]=0$. Write $\mathcal{K}_{t T}=\left(k_{2} / S_{T}\right)^{1 / 2} \mathcal{L}_{t T},(t=1, \ldots, T)$. First, note that

$$
\begin{aligned}
\mathrm{E}^{*}\left[\left|\mathcal{K}_{t_{s}^{*} T}\right|\right] & =\frac{1}{T} \sum_{t=1}^{T}\left|\mathcal{K}_{t T}\right|=\frac{1}{T} \sum_{t=1}^{T}\left|\frac{1}{S_{T}} \sum_{s=t-T}^{t-1} k\left(\frac{s}{S_{T}}\right) \mathcal{L}_{t-s}\right| \\
& \leq O(1) \frac{1}{T} \sum_{t=1}^{T}\left|\mathcal{L}_{t}\right|=O_{p}(1)
\end{aligned}
$$

uniformly, $\left(s=1, \ldots, m_{T}\right)$, by WLLN and $\mathrm{E}\left[\sup _{\theta \in \Theta}\left|\log f_{t}(\theta)\right|^{\alpha}\right]<\infty, \alpha>1$. Also, for any $\delta>0$,

$$
\begin{aligned}
\frac{1}{T} \sum_{t=1}^{T}\left|\mathcal{K}_{t T}\right|-\frac{1}{T} \sum_{t=1}^{T}\left|\mathcal{K}_{t T}\right| \mathbb{I}\left(\left|\mathcal{K}_{t T}\right|\right. & \left.<m_{T} \delta\right)=\frac{1}{T} \sum_{t=1}^{T}\left|\mathcal{K}_{t T}\right| \mathbb{I}\left(\left|\mathcal{K}_{t T}\right| \geq m_{T} \delta\right) \\
& \leq \frac{1}{T} \sum_{t=1}^{T}\left|\mathcal{K}_{t T}\right| \max _{t} \mathbb{I}\left(\left|\mathcal{K}_{t T}\right| \geq m_{T} \delta\right)
\end{aligned}
$$

Now, by M,

$$
\max _{t}\left|\mathcal{K}_{t T}\right|=O(1) \max _{t}\left|\mathcal{L}_{t}\right|=O_{p}\left(T^{1 / \alpha}\right)
$$

cf. Newey and Smith (2004, Proof of Lemma A1, p.239). Hence, since, by hypothesis, $T^{1 / \alpha} / m_{T}=o(1), \max _{t} \mathbb{I}\left(\left|\mathcal{K}_{t T}\right| \geq m_{T} \delta\right)=o_{p}(1)$ and $\sum_{t=1}^{T}\left|\mathcal{K}_{t T}\right| / T=O_{p}(1)$,

$$
\mathrm{E}^{*}\left[\left|\mathcal{K}_{t_{s}^{*} T}\right| \mathbb{I}\left(\left|\mathcal{K}_{t_{s}^{*} T}\right| \geq m_{T} \delta\right)\right]=\frac{1}{T} \sum_{t=1}^{T}\left|\mathcal{K}_{t T}\right| \mathbb{I}\left(\left|\mathcal{K}_{t T}\right| \geq m_{T} \delta\right)=o_{p}(1) .
$$

The remaining part of the proof is similar to that for Khinchine's WLLN given in Rao (1973, pp.112-114). For each $s$ define the pair of random variables

$$
V_{t_{s}^{*} T}=\mathcal{K}_{t_{s}^{*} T} \mathbb{I}\left(\left|\mathcal{K}_{t_{s}^{*} T}\right|<m_{T} \delta\right), W_{t_{s}^{*} T}=\mathcal{K}_{t_{s}^{*} T} \mathbb{I}\left(\left|\mathcal{K}_{t_{s}^{*} T}\right| \geq m_{T} \delta\right)
$$


yielding $\mathcal{K}_{t_{s}^{*} T}=V_{t_{s}^{*} T}+W_{t_{s}^{*} T},\left(s=1, \ldots, m_{T}\right)$. Now

$$
\operatorname{var}^{*}\left[V_{t_{s}^{*} T}\right] \leq \mathrm{E}^{*}\left[V_{t_{s}^{*} T}^{2}\right] \leq m_{T} \delta \mathrm{E}^{*}\left[\left|V_{t_{s}^{*} T}\right|\right]
$$

Write $\bar{V}_{m_{T}}^{*}=\sum_{s=1}^{m_{T}} V_{t_{s}^{*} T} / m_{T}$. Thus, from eq. (A.5), using C,

$$
\begin{aligned}
\mathcal{P}_{\omega}^{*}\left\{\mid \bar{V}_{m_{T}}^{*}-\mathrm{E}^{*}\left[V_{t_{s}^{*} T} T\right]\right. & \geq \varepsilon\} \leq \frac{\operatorname{var}^{*}\left[V_{t_{s}^{*} T}\right]}{m_{T} \varepsilon^{2}} \\
& \leq \frac{\delta \mathrm{E}^{*}\left[\left|V_{t_{s}^{*} T}^{*}\right|\right]}{\varepsilon^{2}} .
\end{aligned}
$$

Also $\left|\overline{\mathcal{K}}_{T}-\mathrm{E}^{*}\left[V_{t_{s}^{*} T}\right]\right|=o_{p}(1)$, i.e., for any $\varepsilon>0$, $T$ large enough, $\left|\overline{\mathcal{K}}_{T}-\mathrm{E}^{*}\left[V_{t_{s}^{*} T}\right]\right| \leq \varepsilon$, since by $\mathrm{T}$, noting $\mathrm{E}^{*}\left[V_{t_{s}^{*} T}\right]=\sum_{t=1}^{T} \mathcal{K}_{t T} \mathbb{I}\left(\left|\mathcal{K}_{t T}\right|<m_{T} \delta\right) / T$,

$$
\begin{aligned}
\left|\overline{\mathcal{K}}_{T}-\mathrm{E}^{*}\left[V_{t_{s}^{*} T}\right]\right| & =\left|\frac{1}{T} \sum_{t=1}^{T} \mathcal{K}_{t T}-\frac{1}{T} \sum_{t=1}^{T} \mathcal{K}_{t T} \mathbb{I}\left(\left|\mathcal{K}_{t T}\right|<m_{T} \delta\right)\right| \\
& \leq \frac{1}{T} \sum_{t=1}^{T}\left|\mathcal{K}_{t T}\right| \mathbb{I}\left(\left|\mathcal{K}_{t T}\right| \geq m_{T} \delta\right)=o_{p}(1)
\end{aligned}
$$

from eq. (A.4). Hence, for $T$ large enough,

$$
\mathcal{P}_{\omega}^{*}\left\{\left|\bar{V}_{m_{T}}^{*}-\overline{\mathcal{K}}_{T}\right| \geq 2 \varepsilon\right\} \leq \frac{\delta \mathrm{E}^{*}\left[\left|V_{t_{s}^{*} T}\right|\right]}{\varepsilon^{2}} .
$$

By M,

$$
\begin{aligned}
\mathcal{P}_{\omega}^{*}\left\{W_{t_{s}^{*} T}\right. & \neq 0\}=\mathcal{P}_{\omega}^{*}\left\{\left|\mathcal{K}_{t_{s}^{*} T}\right| \geq m_{T} \delta\right\} \\
& \leq \frac{1}{m_{T} \delta} \mathrm{E}^{*}\left[\left|\mathcal{K}_{t_{s}^{*} T}\right| \mathbb{I}\left(\left|\mathcal{K}_{t_{s}^{*} T}\right| \geq m_{T} \delta\right)\right] \leq \frac{\delta}{m_{T}} .
\end{aligned}
$$

To see this, $\mathrm{E}^{*}\left[\left|\mathcal{K}_{t_{s}^{*} T}\right| \mathbb{I}\left(\left|\mathcal{K}_{t_{s}^{*} T}\right| \geq m_{T} \delta\right)\right]=o_{p}(1)$ from eq. (A.4). Thus, for $T$ large enough, $\mathrm{E}^{*}\left[\left|\mathcal{K}_{t_{s}^{*} T}\right| \mathbb{I}\left(\left|\mathcal{K}_{t_{s}^{*} T}\right| \geq m_{T} \delta\right)\right] \leq \delta^{2}$ w.p.a.1. Write $\bar{W}_{m_{T}}^{*}=\sum_{s=1}^{m_{T}} W_{t_{s}^{*} T} / m_{T}$. Thus, from eq. (A.7),

$$
\mathcal{P}_{\omega}^{*}\left\{\bar{W}_{m_{T}}^{*} \neq 0\right\} \leq \sum_{s=1}^{m_{T}} \mathcal{P}_{\omega}^{*}\left\{W_{t_{s}^{*} T} \neq 0\right\} \leq \delta
$$

Therefore,

$$
\begin{aligned}
\mathcal{P}_{\omega}^{*}\left\{\left|\overline{\mathcal{K}}_{m_{T}}^{*}-\overline{\mathcal{K}}_{T}\right|\right. & \geq 4 \varepsilon\} \leq \mathcal{P}_{\omega}^{*}\left\{\left|\bar{V}_{m_{T}}^{*}-\overline{\mathcal{K}}_{T}\right|+\left|\bar{W}_{m_{T}}^{*}\right| \geq 4 \varepsilon\right\} \\
& \leq \mathcal{P}_{\omega}^{*}\left\{\left|\bar{V}_{m_{T}}^{*}-\overline{\mathcal{K}}_{T}\right| \geq 2 \varepsilon\right\}+\mathcal{P}_{\omega}^{*}\left\{\left|\bar{W}_{m_{T}}^{*}\right| \geq 2 \varepsilon\right\} \\
& \leq \frac{\delta E^{*}\left[\left|\bar{V}_{t_{s}^{*} T}^{*}\right|\right]}{\varepsilon^{2}}+\mathcal{P}_{\omega}^{*}\left\{\left|\bar{W}_{m_{T}}^{*}\right| \neq 0\right\} \leq \frac{\delta E^{*}\left[\left|V_{t_{s}^{*} T}\right|\right]}{\varepsilon^{2}}+\delta .
\end{aligned}
$$


where the first inequality follows from T, the third from eq. (A.6) and the final inequality from eq. (A.8). Since $\delta$ may be chosen arbitrarily small enough and $\mathrm{E}^{*}\left[\left|V_{t_{s}^{*} T}\right|\right] \leq$ $\mathrm{E}^{*}\left[\left|\mathcal{K}_{t_{s}^{*} T}\right|\right]=O_{p}(1)$, the result follows by M.

Lemma A.3. (Bootstrap CLT.) Let Assumptions 3.2(a)(b), 3.3, 3.4 and 3.6(b)(c) hold. Then, if $S_{T} \rightarrow \infty$ and $S_{T}=O\left(T^{\frac{1}{2}-\eta}\right)$ with $0<\eta<\frac{1}{2}$,

$$
\sup _{x \in \mathcal{R}}\left|\mathcal{P}_{\omega}^{*}\left\{m_{T}^{1 / 2}\left(\frac{\partial \overline{\mathcal{L}}_{m_{T}}^{*}\left(\theta_{0}\right)}{\partial \theta}-\frac{\partial \overline{\mathcal{L}}_{T}\left(\theta_{0}\right)}{\partial \theta}\right) \leq x\right\}-\mathcal{P}\left\{T^{1 / 2} \frac{\partial \overline{\mathcal{L}}\left(\theta_{0}\right)}{\partial \theta} \leq x\right\}\right| \rightarrow 0, \text { prob- } \mathcal{P} \text {. }
$$

Proof. The result is proven in Steps 1-5 below; cf. Politis and Romano (1992b, Proof of Theorem 2, pp. 1994-1995). To ease exposition, let $m_{T}=T / S_{T}$ be integer and $d_{\theta}=1$.

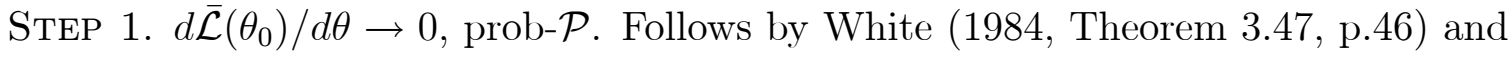
$\mathrm{E}\left[\partial \mathcal{L}_{t}\left(\theta_{0}\right) / \partial \theta\right]=0$.

STEP 2. $\mathcal{P}\left\{B_{0}^{-1 / 2} T^{1 / 2} d \overline{\mathcal{L}}\left(\theta_{0}\right) / d \theta \leq x\right\} \rightarrow \Phi(x)$, where $\Phi(\cdot)$ is the standard normal distribution function. Follows by White (1984, Theorem 5.19, p.124).

STEP 3. $\sup _{x}\left|\mathcal{P}\left\{B_{0}^{-1 / 2} T^{1 / 2} d \overline{\mathcal{L}}\left(\theta_{0}\right) / d \theta \leq x\right\}-\Phi(x)\right| \rightarrow 0$. Follows by Pólya's Theorem (Serfling, 1980, Theorem 1.5.3, p.18) from Step 2 and the continuity of $\Phi(\cdot)$.

STEP 4. $\operatorname{var}^{*}\left[m_{T}^{1 / 2} d \overline{\mathcal{L}}_{m_{T}}^{*}\left(\theta_{0}\right) / d \theta\right] \rightarrow B_{0}$, prob-P. Note $\mathrm{E}^{*}\left[d \overline{\mathcal{L}}_{m_{T}}^{*}\left(\theta_{0}\right) / d \theta\right]=d \overline{\mathcal{L}}_{T}\left(\theta_{0}\right) / d \theta$. Thus,

$$
\begin{aligned}
\operatorname{var}^{*}\left[m_{T}^{1 / 2} \frac{d \overline{\mathcal{L}}_{m_{T}}^{*}\left(\theta_{0}\right)}{d \theta}\right] & =\operatorname{var}^{*}\left[\frac{d \mathcal{L}_{t^{*} T}\left(\theta_{0}\right)}{d \theta}\right] \\
& =\frac{1}{T} \sum_{t=1}^{T}\left(\frac{d \mathcal{L}_{t T}\left(\theta_{0}\right)}{d \theta}-\frac{d \overline{\mathcal{L}}_{T}\left(\theta_{0}\right)}{d \theta}\right)^{2} \\
& =\frac{1}{T} \sum_{t=1}^{T}\left(\frac{d \mathcal{L}_{t T}\left(\theta_{0}\right)}{d \theta}\right)^{2}-\left(\frac{d \overline{\mathcal{L}}_{T}\left(\theta_{0}\right)}{d \theta}\right)^{2}
\end{aligned}
$$

the result follows since $\left(d \overline{\mathcal{L}}_{T}\left(\theta_{0}\right) / d \theta\right)^{2}=O_{p}\left(S_{T} / T\right)$ (Smith, 2011, Lemma A.2, p.1219), $S_{T}=o\left(T^{1 / 2}\right)$ by hypothesis and $T^{-1} \sum_{t=1}^{T}\left(d \mathcal{L}_{t T}\left(\theta_{0}\right) / d \theta\right)^{2} \rightarrow B_{0}$, prob- $\mathcal{P}$ (Smith, 2011, Lemma A.3, p.1219).

STEP 5.

$$
\lim _{T \rightarrow \infty} \mathcal{P}\left\{\sup _{x}\left|\mathcal{P}_{\omega}^{*}\left\{\frac{d \overline{\mathcal{L}}_{m_{T}}^{*}\left(\theta_{0}\right) / d \theta-\mathrm{E}^{*}\left[d \overline{\mathcal{L}}_{m_{T}}^{*}\left(\theta_{0}\right) / d \theta\right]}{\operatorname{var}^{*}\left[d \overline{\mathcal{L}}_{m_{T}}^{*}\left(\theta_{0}\right) / d \theta\right]^{1 / 2}} \leq x\right\}-\Phi(x)\right| \geq \varepsilon\right\}=0 .
$$

Applying the Berry-Esséen inequality, Serfling (1980, Theorem 1.9.5, p.33), noting the bootstrap sample observations $\left\{d \mathcal{L}_{t_{s}^{*} T}\left(\theta_{0}\right) / d \theta\right\}_{s=1}^{m_{T}}$ are independent and identically dis- 
tributed,

$$
\begin{aligned}
\sup _{x} \mid \mathcal{P}_{\omega}^{*}\left\{\frac{m_{T}^{1 / 2}\left(d \overline{\mathcal{L}}_{m_{T}}^{*}\left(\theta_{0}\right) / d \theta-d \overline{\mathcal{L}}_{T}\left(\theta_{0}\right) / d \theta\right)}{\left.\operatorname{var}^{*}\left[m_{T}^{1 / 2} d \overline{\mathcal{L}}_{m_{T}}^{*}\left(\theta_{0}\right) / d \theta\right]^{1 / 2} \leq x\right\}-\Phi(x) \mid \leq}\right. & \frac{C}{m_{T}^{1 / 2}} \operatorname{var}^{*}\left[\frac{d \mathcal{L}_{t^{*} T}\left(\theta_{0}\right)}{d \theta}\right]^{-3 / 2} \\
& \times \mathrm{E}^{*}\left[\left|\frac{d \mathcal{L}_{t^{*} T}\left(\theta_{0}\right)}{d \theta}-\frac{d \overline{\mathcal{L}}_{T}\left(\theta_{0}\right)}{d \theta}\right|^{3}\right] .
\end{aligned}
$$

Now $\operatorname{var}^{*}\left[d \mathcal{L}_{t^{*} T}\left(\theta_{0}\right) / d \theta\right] \rightarrow B_{0}>0$, prob- $\mathcal{P}$; see the Proof of Step 4 above. Furthermore, $\mathrm{E}^{*}\left[\left|d \mathcal{L}_{t^{*} T}\left(\theta_{0}\right) / d \theta-d \overline{\mathcal{L}}_{T}\left(\theta_{0}\right) / d \theta\right|^{3}\right]=T^{-1} \sum_{t=1}^{T}\left|d \mathcal{L}_{t T}\left(\theta_{0}\right) / d \theta-d \overline{\mathcal{L}}_{T}\left(\theta_{0}\right) / d \theta\right|^{3}$ and

$$
\begin{aligned}
\frac{1}{T} \sum_{t=1}^{T}\left|\frac{d \mathcal{L}_{t T}\left(\theta_{0}\right)}{d \theta}-\frac{d \overline{\mathcal{L}}_{T}\left(\theta_{0}\right)}{d \theta}\right|^{3} & \leq \max _{t}\left|\frac{d \mathcal{L}_{t T}\left(\theta_{0}\right)}{d \theta}-\frac{d \overline{\mathcal{L}}_{T}\left(\theta_{0}\right)}{d \theta}\right| \frac{1}{T} \sum_{t=1}^{T}\left(\frac{d \mathcal{L}_{t T}\left(\theta_{0}\right)}{d \theta}-\frac{d \overline{\mathcal{L}}_{T}\left(\theta_{0}\right)}{d \theta}\right)^{2} \\
& =O_{p}\left(S_{T}^{1 / 2} T^{1 / \alpha}\right) .
\end{aligned}
$$

The equality follows since

$$
\begin{aligned}
\max _{t}\left|\frac{d \mathcal{L}_{t T}\left(\theta_{0}\right)}{d \theta}-\frac{d \overline{\mathcal{L}}_{T}\left(\theta_{0}\right)}{d \theta}\right| & \leq \max _{t}\left|\frac{d \mathcal{L}_{t T}\left(\theta_{0}\right)}{d \theta}\right|+\left|\frac{d \overline{\mathcal{L}}_{T}\left(\theta_{0}\right)}{d \theta}\right| \\
& =O_{p}\left(S_{T}^{1 / 2} T^{1 / \alpha}\right)+O_{p}\left(\left(S_{T} / T\right)^{1 / 2}\right)=O_{p}\left(S_{T}^{1 / 2} T^{1 / \alpha}\right)
\end{aligned}
$$

by $\mathrm{M}$ and Assumption 3.6(b), cf. Newey and Smith (2004, Proof of Lemma A1, p.239), and $\sum_{t=1}^{T}\left(d \mathcal{L}_{t T}\left(\theta_{0}\right) / d \theta-d \overline{\mathcal{L}}_{T}\left(\theta_{0}\right) / d \theta\right)^{2} / T=O_{p}(1)$, see the Proof of Step 4 above. Therefore

$$
\begin{aligned}
\sup _{x}\left|\mathcal{P}_{\omega}^{*}\left\{\frac{\left(T / S_{T}\right)^{1 / 2}\left(d \overline{\mathcal{L}}_{m_{T}}^{*}\left(\theta_{0}\right) / d \theta-d \overline{\mathcal{L}}_{T}\left(\theta_{0}\right) / d \theta\right)}{\operatorname{var}^{*}\left[\left(T / S_{T}\right)^{1 / 2} d \overline{\mathcal{L}}_{m_{T}}^{*}\left(\theta_{0}\right) / d \theta\right]^{1 / 2}} \leq x\right\}-\Phi(x)\right| & \leq \frac{1}{m_{T}^{1 / 2}} O_{p}(1) O_{p}\left(S_{T}^{1 / 2} T^{1 / \alpha}\right) \\
& =\frac{S_{T}^{1 / 2}}{m_{T}^{1 / 2}} O_{p}\left(T^{1 / \alpha}\right)=o_{p}(1),
\end{aligned}
$$

by hypothesis, yielding the required conclusion.

Lemma A.4. Suppose that Assumptions 3.2(a)(b), 3.3, 3.4 and 3.6(b)(c) hold. Then, if $S_{T} \rightarrow \infty$ and $S_{T}=O\left(T^{\frac{1}{2}-\eta}\right)$ with $0<\eta<\frac{1}{2}$,

$$
\left(k_{2} / S_{T}\right)^{1 / 2} \frac{\partial \overline{\mathcal{L}}_{T}\left(\theta_{0}\right)}{\partial \theta}=k_{1} \frac{\partial \overline{\mathcal{L}}\left(\theta_{0}\right)}{\partial \theta}+o_{p}\left(T^{-1 / 2}\right) .
$$

Proof. Cf. Smith (2011, Proof of Lemma A.2, p.1219). Recall

$$
\left(k_{2} / S_{T}\right)^{1 / 2} \frac{\partial \overline{\mathcal{L}}_{T}\left(\theta_{0}\right)}{\partial \theta}=\frac{1}{S_{T}} \sum_{r=1-T}^{T-1} k\left(\frac{r}{S_{T}}\right) \frac{1}{T} \sum_{t=\max [1,1-r]}^{\min [T, T-r]} \frac{\partial \mathcal{L}_{t}\left(\theta_{0}\right)}{\partial \theta} .
$$


The difference between $\sum_{t=\max [1,1-r]}^{\min [T, T-r]} \partial \mathcal{L}_{t}\left(\theta_{0}\right) / \partial \theta$ and $\sum_{t=1}^{T} \partial \mathcal{L}_{t}\left(\theta_{0}\right) / \partial \theta$ consists of $|r|$ terms. By C, using White (1984, Lemma 6.19, p.153),

$$
\begin{aligned}
\mathcal{P}\left\{\frac{1}{T}\left|\sum_{t=1}^{|r|} \frac{\partial \mathcal{L}_{t}\left(\theta_{0}\right)}{\partial \theta}\right|\right. & \geq \varepsilon\} \leq \frac{1}{(T \varepsilon)^{2}} \mathrm{E}\left[\left|\sum_{t=1}^{|r|} \frac{\partial \mathcal{L}_{t}\left(\theta_{0}\right)}{\partial \theta}\right|^{2}\right] \\
& =|r| O\left(T^{-2}\right)
\end{aligned}
$$

where the $O\left(T^{-2}\right)$ term is independent of $r$. Therefore, using Smith (2011, Lemma C.1, p.1231),

$$
\begin{aligned}
\left(k_{2} / S_{T}\right)^{1 / 2} \frac{\partial \overline{\mathcal{L}}_{T}\left(\theta_{0}\right)}{\partial \theta} & =\frac{1}{S_{T}} \sum_{r=1-T}^{T-1} k\left(\frac{r}{S_{T}}\right)\left(\frac{\partial \overline{\mathcal{L}}\left(\theta_{0}\right)}{\partial \theta}+|r| O_{p}\left(T^{-2}\right)\right) \\
& =\frac{1}{S_{T}} \sum_{s=1-T}^{T-1} k\left(\frac{s}{S_{T}}\right) \frac{\partial \overline{\mathcal{L}}\left(\theta_{0}\right)}{\partial \theta}+O_{p}\left(T^{-1}\right) \\
& =\left(k_{1}+o(1)\right) \frac{\partial \overline{\mathcal{L}}\left(\theta_{0}\right)}{\partial \theta}+O_{p}\left(T^{-1}\right) \\
& =k_{1} \frac{\partial \overline{\mathcal{L}}\left(\theta_{0}\right)}{\partial \theta}+o_{p}\left(T^{-1 / 2}\right)
\end{aligned}
$$

\section{Appendix B: Proofs of Results}

Proof of Theorem 3.1. Theorem 3.1 follows from a verification of the hypotheses of Gonçalves and White (2004, Lemma A.2, p.212). To do so, replace $n$ by $T, Q_{T}(\cdot, \theta)$ by $\overline{\mathcal{L}}(\theta)$ and $Q_{T}^{*}(\cdot, \omega, \theta)$ by $\overline{\mathcal{L}}_{m_{T}}^{*}(\omega, \theta)$. Conditions (a1)-(a3), which ensure $\hat{\theta}-\theta_{0} \rightarrow 0$, prob- $\mathcal{P}$, hold under Assumptions 3.1 and 3.2. To establish $\hat{\theta}^{*}-\hat{\theta} \rightarrow 0$, prob- $\mathcal{P}_{\omega}^{*}$, prob$\mathcal{P}$, Conditions (b1) and (b2) follow from Assumption 3.1 whereas Condition (b3) is the bootstrap UWL Lemma A.1 which requires Assumption 3.3.

Proof of Theorem 3.2. The structure of the proof is identical to that of Gonçalves and White (2004, Theorem 2.2, pp.213-214) for MBB requiring the verification of the hypotheses of Gonçalves and White (2004, Lemma A.3, p.212) which together with Pólya's Theorem, Serfling (1980, Theorem 1.5.3, p.18), and the continuity of $\Phi(\cdot)$ gives the result.

Assumptions 3.2-3.4 ensure Theorem 3.1, i.e., $\hat{\theta}^{*}-\hat{\theta} \rightarrow 0$, prob- $\mathcal{P}_{\omega}^{*}$, prob- $\mathcal{P}$, and $\hat{\theta}-\theta_{0} \rightarrow 0$, prob- $\mathcal{P}$. The assumptions of the complete probability space $(\Omega, \mathcal{F}, \mathcal{P})$ and compactness of $\Theta$ are stated in Assumptions 3.4(a) and 3.5(a). Conditions (a1) and (a2) follow from Assumptions $3.5(\mathrm{a})(\mathrm{b})$. Condition $(\mathrm{a} 3) B_{0}^{-1 / 2} T^{1 / 2} \partial \overline{\mathcal{L}}\left(\theta_{0}\right) / \partial \theta \stackrel{d}{\rightarrow} N\left(0, I_{d_{\theta}}\right)$ is satisfied under Assumptions 3.4, 3.5(a)(b) and 3.6(b)(c) using the CLT White (1984, 
Theorem 5.19, p.124); cf. Step 4 in the Proof of Lemma A.3 above. The continuity of $A(\theta)$ and the UWL Condition $(\mathrm{a} 4) \sup _{\theta \in \Theta}\left\|\partial^{2} \overline{\mathcal{L}}(\theta) / \partial \theta \partial \theta^{\prime}-A(\theta)\right\| \rightarrow 0$, prob- $\mathcal{P}$, follow since the hypotheses of the UWL Newey and McFadden (1994, Lemma 2.4, p.2129) for stationary and mixing (and, thus, ergodic) processes are satisfied under Assumptions 3.43.6. Hence, invoking Assumption 3.6(c), from a mean value expansion of $\partial \overline{\mathcal{L}}(\hat{\theta}) / \partial \theta=0$ around $\theta=\theta_{0}$ with $\theta_{0} \in \operatorname{int}(\Theta)$ from Assumption $3.5(\mathrm{c}), T^{1 / 2}\left(\hat{\theta}-\theta_{0}\right) \stackrel{d}{\rightarrow} N\left(0, A_{0}^{-1} B_{0} A_{0}^{-1}\right)$.

Conditions (b1) and (b2) are satisfied under Assumptions 3.5(a)(b) as above. To verify Condition (b3),

$$
\begin{aligned}
m_{T}^{1 / 2} \frac{\partial \overline{\mathcal{L}}_{m_{T}}^{*}(\hat{\theta})}{\partial \theta}= & m_{T}^{1 / 2}\left(\frac{\partial \overline{\mathcal{L}}_{m_{T}}^{*}\left(\theta_{0}\right)}{\partial \theta}-\frac{\partial \overline{\mathcal{L}}_{T}\left(\theta_{0}\right)}{\partial \theta}\right) \\
& +m_{T}^{1 / 2} \frac{\partial \overline{\mathcal{L}}_{T}\left(\theta_{0}\right)}{\partial \theta}+m_{T}^{1 / 2}\left(\frac{\partial \overline{\mathcal{L}}_{m_{T}}^{*}(\hat{\theta})}{\partial \theta}-\frac{\partial \overline{\mathcal{L}}_{m_{T}}^{*}\left(\theta_{0}\right)}{\partial \theta}\right) .
\end{aligned}
$$

With Lemma A.3 replacing Gonçalves and White (2002, Theorem 2.2(ii), p.1375), the first term converges in distribution to $N\left(0, B_{0}\right)$, prob- $\mathcal{P}_{\omega}^{*}$, prob- $\mathcal{P}$. The sum of the second and third terms converges to 0 , prob- $\mathcal{P}_{\omega}^{*}$, prob- $\mathcal{P}$. To see this, first, using the mean value theorem for the third term, i.e.,

$$
m_{T}^{1 / 2}\left(\frac{\partial \overline{\mathcal{L}}_{m_{T}}^{*}(\hat{\theta})}{\partial \theta}-\frac{\partial \overline{\mathcal{L}}_{m_{T}}^{*}\left(\theta_{0}\right)}{\partial \theta}\right)=\frac{1}{S_{T}^{1 / 2}} \frac{\partial^{2} \overline{\mathcal{L}}_{m_{T}}^{*}(\dot{\theta})}{\partial \theta \partial \theta^{\prime}} T^{1 / 2}\left(\hat{\theta}-\theta_{0}\right),
$$

where $\dot{\theta}$ lies on the line segment joining $\hat{\theta}$ and $\theta_{0}$. Secondly, $\left(k_{2} / S_{T}\right)^{1 / 2} \partial^{2} \overline{\mathcal{L}}_{m_{T}}^{*}(\dot{\theta}) / \partial \theta \partial \theta^{\prime} \rightarrow$ $k_{1} A_{0}$, prob- $\mathcal{P}_{\omega}^{*}$, prob- $\mathcal{P}$, using the bootstrap UWL $\sup _{\theta \in \Theta}\left(k_{2} / S_{T}\right)^{1 / 2}\left\|\partial^{2} \overline{\mathcal{L}}_{m_{T}}^{*}(\theta) / \partial \theta \partial \theta^{\prime}-\partial^{2} \overline{\mathcal{L}}_{T}(\theta) / \partial \theta \partial \theta^{\prime}\right\|$ $\rightarrow 0$, prob- $\mathcal{P}_{\omega}^{*}$, prob- $\mathcal{P}$, cf. Lemma A.1, and the UWL $\sup _{\theta \in \Theta}\left\|\left(k_{2} / S_{T}\right)^{1 / 2} \partial^{2} \overline{\mathcal{L}}_{T}(\theta) / \partial \theta \partial \theta^{\prime}-k_{1} A(\theta)\right\| \rightarrow$ 0 , prob- $\mathcal{P}$, cf. Remark A.2. Condition (b3) then follows since $T^{1 / 2}\left(\hat{\theta}-\theta_{0}\right)+A_{0}^{-1} T^{1 / 2} \partial \overline{\mathcal{L}}\left(\theta_{0}\right) / \partial \theta \rightarrow$ 0 , prob- $\mathcal{P}$, and $m_{T}^{1 / 2} \partial \overline{\mathcal{L}}_{T}\left(\theta_{0}\right) / \partial \theta-\left(k_{1} / k_{2}^{1 / 2}\right) T^{1 / 2} \partial \overline{\mathcal{L}}\left(\theta_{0}\right) / \partial \theta \rightarrow 0$, prob- $\mathcal{P}$, cf. Lemma A.4. Finally, Condition (b4) $\sup _{\theta \in \Theta}\left\|\left(k_{2} / S_{T}\right)^{1 / 2}\left[\partial^{2} \overline{\mathcal{L}}_{m_{T}}^{*}(\theta) / \partial \theta \partial \theta^{\prime}-\partial^{2} \overline{\mathcal{L}}_{T}(\theta) / \partial \theta \partial \theta^{\prime}\right]\right\| \rightarrow$ 0 , prob- $\mathcal{P}_{\omega}^{*}$, prob- $\mathcal{P}$, is the bootstrap UWL Lemma A.1 appropriately revised using Assumption 3.6.

Because $\hat{\theta} \in \operatorname{int}(\Theta)$ from Assumption 3.5(c), from a mean value expansion of the first order condition $\partial \overline{\mathcal{L}}_{m_{T}}^{*}\left(\hat{\theta}^{*}\right) / \partial \theta=0$ around $\theta=\hat{\theta}$,

$$
T^{1 / 2}\left(\hat{\theta}^{*}-\hat{\theta}\right)=\left[\frac{\partial^{2} \overline{\mathcal{L}}_{m_{T}}^{*}(\dot{\theta})}{\partial \theta \partial \theta^{\prime}} / S_{T}^{1 / 2}\right]^{-1} m_{T}^{1 / 2} \frac{\partial \overline{\mathcal{L}}_{m_{T}}^{*}(\hat{\theta})}{\partial \theta},
$$

where $\dot{\theta}$ lies on the line segment joining $\hat{\theta}^{*}$ and $\hat{\theta}$. Noting $\hat{\theta}^{*}-\hat{\theta} \rightarrow 0$, prob- $\mathcal{P}_{\omega}^{*}$, prob- $\mathcal{P}$, and $\hat{\theta}-\theta_{0} \rightarrow 0$, prob- $\mathcal{P},\left(k_{2} / S_{T}\right)^{1 / 2} \partial^{2} \overline{\mathcal{L}}_{m_{T}}^{*}(\dot{\theta}) / \partial \theta \partial \theta^{\prime} \rightarrow k_{1} A_{0}$, prob- $\mathcal{P}_{\omega}^{*}$, prob- $\mathcal{P}$. Therefore, $T^{1 / 2}\left(\hat{\theta}^{*}-\hat{\theta}\right)$ converges in distribution to $N\left(0,\left(k_{2} / k_{1}^{2}\right) A_{0}^{-1} B_{0} A_{0}^{-1}\right)$, prob- $\mathcal{P}_{\omega}^{*}$, prob- $\mathcal{P}$. 
Proof of Corollary 3.1. It follows immediately from Lemma A.3 that

$$
m_{T}^{1 / 2}\left(\frac{\partial \overline{\mathcal{L}}_{m_{T}}^{*}\left(\theta_{0}\right)}{\partial \theta}-\frac{\partial \overline{\mathcal{L}}_{T}\left(\theta_{0}\right)}{\partial \theta}\right) \stackrel{d}{\rightarrow} N\left(0, B_{0}\right), \text { prob- } \mathcal{P}_{\omega}^{*}, \text { prob- } \mathcal{P} .
$$

Moreover, from the Proof of Theorem 3.2,

$$
m_{T}^{1 / 2} \frac{\partial \overline{\mathcal{L}}_{m_{T}}^{*}(\hat{\theta})}{\partial \theta}-m_{T}^{1 / 2}\left(\frac{\partial \overline{\mathcal{L}}_{m_{T}}^{*}\left(\theta_{0}\right)}{\partial \theta}-\frac{\partial \overline{\mathcal{L}}_{T}\left(\theta_{0}\right)}{\partial \theta}\right) \rightarrow 0, \text { prob- } \mathcal{P}_{\omega}^{*}, \text { prob- } \mathcal{P} .
$$

Therefore,

$$
\sup _{x \in \mathcal{R}^{d_{\theta}}}\left|\mathcal{P}_{\omega}^{*}\left\{m_{T}^{1 / 2} \frac{\partial \overline{\mathcal{L}}_{m_{T}}^{*}(\hat{\theta})}{\partial \theta} \leq x\right\}-\mathcal{P}\left\{T^{1 / 2} \frac{\partial \overline{\mathcal{L}}\left(\theta_{0}\right)}{\partial \theta} \leq x\right\}\right| \rightarrow 0, \text { prob-P }
$$

follows by Pólya's Theorem, Serfling (1980, Theorem 1.5.3, p.18) and the continuity of the normal c.d.f. $\Phi(\cdot)$ recalling $\sup _{x}\left|\mathcal{P}\left\{B_{0}^{-1 / 2} T^{1 / 2} d \overline{\mathcal{L}}\left(\theta_{0}\right) / d \theta \leq x\right\}-\Phi(x)\right| \rightarrow 0$ from SteP 3 of the Proof of Lemma A.3.

Recall from eq. (B.1) in the Proof of Theorem 3.2 that, because $\hat{\theta} \in \operatorname{int}(\Theta)$, from a mean value expansion of the first order condition $\partial \overline{\mathcal{L}}_{m_{T}}^{*}\left(\hat{\theta}^{*}\right) / \partial \theta=0$ around $\theta=\hat{\theta}$,

$$
T^{1 / 2}\left(\hat{\theta}^{*}-\hat{\theta}\right)=\left[\frac{\partial^{2} \overline{\mathcal{L}}_{m_{T}}^{*}(\dot{\theta})}{\partial \theta \partial \theta^{\prime}} / S_{T}^{1 / 2}\right]^{-1} m_{T}^{1 / 2} \frac{\partial \overline{\mathcal{L}}_{m_{T}}^{*}(\hat{\theta})}{\partial \theta} .
$$

First, $\left(k_{2} / S_{T}\right)^{1 / 2} \partial^{2} \overline{\mathcal{L}}_{m_{T}}^{*}(\dot{\theta}) / \partial \theta \partial \theta^{\prime} \rightarrow k_{1} A_{0}$, prob- $\mathcal{P}_{\omega}^{*}$, prob- $\mathcal{P}$, using the bootstrap UWL $\sup _{\theta \in \Theta}\left(k_{2} / S_{T}\right)^{1 / 2}\left\|\partial^{2} \overline{\mathcal{L}}_{m_{T}}^{*}(\theta) / \partial \theta \partial \theta^{\prime}-\partial^{2} \overline{\mathcal{L}}_{T}(\theta) / \partial \theta \partial \theta^{\prime}\right\| \rightarrow 0$, prob- $\mathcal{P}_{\omega}^{*}$, prob- $\mathcal{P}$, cf. Lemma A.1, and the UWL $\sup _{\theta \in \Theta}\left\|\left(k_{2} / S_{T}\right)^{1 / 2} \partial^{2} \overline{\mathcal{L}}_{T}(\theta) / \partial \theta \partial \theta^{\prime}-k_{1} A(\theta)\right\| \rightarrow 0$, prob- $\mathcal{P}$, cf. Remark A.2. Secondly, similarly, $\left(k_{2} / S_{T}\right)^{1 / 2}\left\|\partial^{2} \overline{\mathcal{L}}_{m_{T}}^{*}(\dot{\theta}) / \partial \theta \partial \theta^{\prime}-\partial^{2} \overline{\mathcal{L}}_{m_{T}}^{*}(\hat{\theta}) / \partial \theta \partial \theta^{\prime}\right\| \rightarrow 0$, prob- $\mathcal{P}_{\omega}^{*}$, prob- $\mathcal{P}$. Hence,

$$
T^{1 / 2}\left(\hat{\theta}^{*}-\hat{\theta}\right)-\left[\frac{\partial^{2} \overline{\mathcal{L}}_{m_{T}}^{*}(\hat{\theta})}{\partial \theta \partial \theta^{\prime}} / S_{T}^{1 / 2}\right]^{-1} m_{T}^{1 / 2} \frac{\partial \overline{\mathcal{L}}_{m_{T}}^{*}(\hat{\theta})}{\partial \theta} \rightarrow 0, \text { prob- } \mathcal{P}_{\omega}^{*}, \text { prob-P } .
$$

Therefore, from Theorem 3.2, after substitution of (B.2),

$$
\sup _{x \in \mathcal{R}^{d_{\theta}}}\left|\mathcal{P}_{\omega}^{*}\left\{\left[\frac{\partial^{2} \overline{\mathcal{L}}_{m_{T}}^{*}(\hat{\theta})}{\partial \theta \partial \theta^{\prime}} / S_{T}^{1 / 2}\right]^{-1} m_{T}^{1 / 2} \frac{\partial \overline{\mathcal{L}}_{m_{T}}^{*}(\hat{\theta})}{\partial \theta} / k^{1 / 2} \leq x\right\}-\mathcal{P}\left\{T^{1 / 2}\left(\hat{\theta}-\theta_{0}\right) \leq x\right\}\right| \rightarrow 0, \text { prob-P },
$$

or

$$
\sup _{x \in \mathcal{R}^{d_{\theta}}}\left|\mathcal{P}_{\omega}^{*}\left\{\left[\frac{\partial^{2} \overline{\mathcal{L}}_{m_{T}}^{*}(\hat{\theta})}{\partial \theta \partial \theta^{\prime}}\right]^{-1} T^{1 / 2} \frac{\partial \overline{\mathcal{L}}_{m_{T}}^{*}(\hat{\theta})}{\partial \theta} / k^{1 / 2} \leq x\right\}-\mathcal{P}\left\{T^{1 / 2}\left(\hat{\theta}-\theta_{0}\right) \leq x\right\}\right| \rightarrow 0, \text { prob-P. }
$$


Proof of Theorem 3.3. Because of its brevity we offer a proof of the limit distribution of the LR statistic; see also White (1994, Theorem 8.10, p. 177). By a second order Taylor expansion of $\overline{\mathcal{L}}\left(\theta_{0}\right)$ around $\hat{\theta}$

$$
2 T\left(\overline{\mathcal{L}}(\hat{\theta})-\overline{\mathcal{L}}\left(\theta_{0}\right)\right)=-T^{1 / 2}\left(\hat{\theta}-\theta_{0}\right)^{\prime} \frac{\partial^{2} \overline{\mathcal{L}}(\dot{\theta})}{\partial \theta \partial \theta^{\prime}} T^{1 / 2}\left(\hat{\theta}-\theta_{0}\right)
$$

where $\dot{\theta}$ lies on the line segment joining $\hat{\theta}$ and $\theta_{0}$. Let $\lambda$ denotes the $d_{\theta}$-vector of eigenvalues of $-B_{0} A_{0}^{-1}$. Since $T^{1 / 2}\left(\hat{\theta}-\theta_{0}\right) \stackrel{d}{\rightarrow} N\left(0, A_{0}^{-1} B_{0} A_{0}^{-1}\right)$ from the Proof of Theorem 3.2 and UWL $\partial^{2} \overline{\mathcal{L}}(\theta) / \partial \theta \partial \theta^{\prime}-A(\theta) \rightarrow 0$, prob- $\mathcal{P}$, cf. Remark A.2, then, noting Vuong (1989, Lemma 3.2, p.312), $2 T\left(\overline{\mathcal{L}}(\hat{\theta})-\overline{\mathcal{L}}\left(\theta_{0}\right)\right) \stackrel{d}{\rightarrow} M_{d_{\theta}}(\cdot, \lambda)$ uniformly follows from Polya's Theorem, Serfling (1980, Theorem 1.5.3, p.18), and the continuity of the c.d.f. $M_{d_{\theta}}(\cdot, \lambda)$.

Similarly, by a second order Taylor expansion of $\overline{\mathcal{L}}_{m_{T}}^{*}(\hat{\theta})$ about $\overline{\mathcal{L}}_{m_{T}}^{*}\left(\hat{\theta}^{*}\right)$, recalling $k=k_{2} / k_{1}^{2}$,

$$
2 \frac{T}{\left(k S_{T}\right)^{1 / 2}}\left(\overline{\mathcal{L}}_{m_{T}}^{*}\left(\hat{\theta}^{*}\right)-\overline{\mathcal{L}}_{m_{T}}^{*}(\hat{\theta})\right)=-\frac{T^{1 / 2}}{k^{1 / 2}}\left(\hat{\theta}^{*}-\hat{\theta}\right)^{\prime}\left(k / S_{T}\right)^{1 / 2} \frac{\partial^{2} \overline{\mathcal{L}}_{m_{T}}^{*}\left(\dot{\theta}^{*}\right)}{\partial \theta \partial \theta^{\prime}} \frac{T^{1 / 2}}{k^{1 / 2}}\left(\hat{\theta}^{*}-\hat{\theta}\right)
$$

where $\dot{\theta}^{*}$ is on the line segment joining $\hat{\theta}^{*}$ and $\hat{\theta}$. Noting $\left(k / S_{T}\right)^{1 / 2} \partial^{2} \overline{\mathcal{L}}_{m_{T}}^{*}\left(\dot{\theta}^{*}\right) / \partial \theta \partial \theta^{\prime} \rightarrow A_{0}$ and $T^{1 / 2}\left(\hat{\theta}^{*}-\hat{\theta}\right) / k^{1 / 2}$ converges in distribution to $N\left(0, A_{0}^{-1} B_{0} A_{0}^{-1}\right)$, prob- $\mathcal{P}_{\omega}^{*}$, prob- $\mathcal{P}$, from the bootstrap UWL Lemma A.1 and Theorem 3.2 respectively, then $2\left(T /\left(k S_{T}\right)^{1 / 2}\right)\left(\overline{\mathcal{L}}_{m_{T}}^{*}\left(\hat{\theta}^{*}\right)-\right.$ $\left.\overline{\mathcal{L}}_{m_{T}}^{*}(\hat{\theta})\right)$ converges in distribution to $\mathcal{M}_{d_{\theta}}(\cdot, \lambda)$, prob- $\mathcal{P}_{\omega}^{*}$, prob- $\mathcal{P}$, and the result follows.

\section{Data Availability Statement}

The data that support the findings of this study are available from the corresponding author upon reasonable request.

\section{REFERENCES}

Andrews, D.W.K. (1991). Heteroskedasticity and Autocorrelation Consistent Covariance Matrix Estimation. Econometrica, 59, 817-858.

Andrews, D.W.K. (2002). Higher-Order Improvements of a Computationally Attractive k-Step Bootstrap for Extremum Estimators. Econometrica, 70, 119-162.

Andrews, D.W.K. (2004). The Block-Block Bootstrap: Improved Asymptotic Refinements. Econometrica, 72, 673-700. 
Andrews, D.W.K., And Monahan, J.C. (1992). An Improved Heteroskedasticity and Autocorrelation Consistent Covariance Matrix Estimator. Econometrica, 60, 1992, 953-966.

Bickel, P., And Freedman, D. (1981). Some Asymptotic Theory of the Bootstrap. Annals of Statistics, 9, 1196-1217.

Brillinger, D.R. (1981). Time Series: Data Analysis and Theory. San Francisco: Holden Day.

Bühlmann, P. (1997). Sieve Bootstrap for Time Series. Bernoulli, 3, 123-148.

Camponovo, L., Scaillet, O., and Trojani, F. (2012). Robust subsampling. Journal of Econometrics, 167, 197-210.

Davison, A. C., And Hall, P. (1993). On Studentizing and Blocking Methods for Implementing the Bootstrap with Dependent Data. Australian Journal of Statistics, 35, 215-224.

Davidson, R., And MacKinnon, J.G. (1999). Bootstrap Testing in Nonlinear Models, International Economic Review, 40, 487-508.

Dufour, J.-M. (2003). Identification, Weak Instruments, and Statistical Iinference in Econometrics. Canadian Journal of Economics, 36, 767-808.

Efron, B. (1979). Bootstrap Methods: Another Look at the Jackknife. Annals of Statistics, 7, 1-26.

Epanechnikov, V.A. (1969). Non-parametric Estimation of a Multivariate Probability Density. Theory of Probability and Its Applications, 14, 153-158.

Fitzenberger, B. (1997). The Moving Blocks Bootstrap and Robust Inference for Linear Least Squares and Quantile Regressions. Journal of Econometrics, 82, 235287.

Gonçalves, S., And White, H. (2000). Maximum Likelihood and the Bootstrap for Nonlinear Dynamic Models. University of California at San Diego, Economics Working Paper Series 2000-32, Department of Economics, UC San Diego.

Gonçalves, S., And White, H. (2002). The Bootstrap of the Mean for Dependent Heterogeneous Arrays. Econometric Theory, 18, 1367-1384. 
Gonçalves, S., And White, H. (2004). Maximum Likelihood and the Bootstrap for Nonlinear Dynamic Models. Journal of Econometrics, 119, 199-219.

Götze, F., AND Künsch, H.R. (1996). Second-Order Correctness of the Blockwise Bootstrap for Stationary Observations. Annals of Statistics, 24, 1914-1933.

Gradshteyn, I.S., And Ryzhik, I.M. (1980). Tables of Integrals, Series and Products. New York: Academic Press.

Hall, P. (1992). The Bootstrap and Edgeworth Expansion. New York: Springer.

Hall, P., AND Horowitz, J. (1996). Bootstrap Critical Values for Tests Based on Generalized-Method-of-Moments Estimators. Econometrica, 64, 891-916.

Hansen, L.P. (1982). Large Sample Properties of Generalized Method of Moments Estimators. Econometrica. 50, 1029-1054.

Hidalgo, J. (2003). An Alternative Bootstrap to Moving Blocks for Time Series Regression Models. Journal of Econometrics, 117, 369-399.

Hong, H., And Scaillet, O. (2006). A Fast Subsampling Method for Nonlinear Dynamic Models. Journal of Econometrics, 133, 557-578.

Horowitz, J.L. (2001). The Bootstrap in Econometrics. Chapter 52 in J.J. Heckman and E.E. Leamer, eds., Handbook of Econometrics, Volume 5, 3159-3228. New York: Elsevier.

Hurvich, C., And Zeger, S. (1987). Frequency Domain Bootstrap Methods for Time Series. Statistics and Operation Research Working Paper, New York University.

Ibragimov, I.A., AND LinniK, Y.V. (1971). Independent and Stationary Sequences of Random Variables. Groningen : Wolters-Noordhoff.

Inoue, A., And Shintani, M. (2006). Bootstrapping GMM Estimators for Time Series. Journal of Econometrics, 133, 531-555.

Kitamura, Y., And Stutzer, M. (1997). An Information-Theoretic Alternative to Generalized Method of Moments Estimation. Econometrica, 65, 861-874.

Kreiss, J.P., And Paparoditis, E. (2011). Bootstrap Methods for Dependent Data: A Review. Journal of the Korean Statistical Society, 40, 357-378. 
Künsch, H.R. (1989). The Jackknife and the Bootstrap for General Stationary Observations. Annals of Statistics, 17, 1217-1241.

La Vecchia, D., Moor A., And Scaillet, O. (2020). A Higher-Order Correct Fast Moving-Average Bootstrap for Dependent Data. Swiss finance Institute DP 2020.01. arXiv:2001.04867v1.

LAhiRI, S.N. (1996). On Edgeworth Expansion and Moving Block Bootstrap for Studentized M-Estimators in Multiple Linear Regression Models. Journal of Multivariate Analysis, 56, 42-59.

LAhiRI, S.N. (2003). Resampling Methods for Dependent Data. New York: Springer.

Liu, R., And Singh, K. (1992). Moving Blocks Jackknife and Bootstrap Capture Weak Dependence. In LePage, R., and Billard L. (Eds.), Exploring the Limits of Bootstrap, 224-248. New York: Wiley.

Magnus, J.R. And Neudecker, H. (1979). The Commutation Matrix: Some Properties and Applications. Annals of Statistics, 7, 381-394.

McMurry, T.L., And Politis, D.N. (2018). Estimating MA Parameters through Factorization of the Autocovariance Matrix and an MA-Sieve Bootstrap. Journal of Time Series Analysis, 39, 433-446.

Newey, W.K., And McFadden, D.L. (1994). Large Sample Estimation and Hypothesis Testing. Chapter 36 in Handbook of Econometrics, Vol. 4, eds. R.F. Engle and D.L. McFadden, 2111-2245. New York: North Holland.

Newey, W.K. And Smith, R.J. (2004). Higher Order Properties of GMM and Generalized Empirical Likelihood Estimators. Econometrica, 72, 219-255.

Nze, P.A., And Doukhan, P. (2004). Weak Dependence: Models and Applications to Econometrics. Econometric Theory, 20, 995-1045.

Paparoditis, E., And Politis, D.N. (2001). Tapered Block Bootstrap. Biometrika, 88, 1105-1119.

Paparoditis, E., And Politis, D.N. (2002). The Tapered Block Bootstrap for General Statistics from Stationary Sequences. Econometrics Journal, 5, 131-148. 
Parente, P.M.D.C., And Smith, R.J. (2019). Kernel Block Bootstrap. Revision of CWP 48/18, Centre for Microdata Methods and Practice, U.C.L and I.F.S.

Parzen, E. (1957). On Consistent Estimates of the Spectrum of a Stationary Time Series. Annals.of Mathematical Statistics, 28, 329-348.

Politis, D.N., and Romano, J. (1992a). A Circular Block-Resampling Procedure for Stationary Data. In LePage, R., and Billard L. (Eds.), Exploring the Limits of Bootstrap, 263-270. New York: Wiley.

Politis, D.N., And Romano, J. (1992b). A General Resampling Scheme for Triangular Arrays of $\alpha$-Mixing Random Variables with Application to the Problem of Spectral Density Estimation. Annals of Statistics, 20, 1985-2007.

Politis, D.N., And Romano, J. (1994). The Stationary Bootstrap. Journal of the American Statististical Association, 89, 1303-1313.

Politis, D.N., And Romano, J. (1995). Bias-Corrected Nonparametric Spectral Estimation. Journal of Time Series Analysis, 16, 68-103.

Politis, D.N., And White, H. (2004). Automatic Block-Length Selection for the Dependent Bootstrap. Econometric Reviews, 23, 53-70.

Priestley, M.B. (1962). Basic Considerations in the Estimation of Spectra. Technometrics, 4, 551-5154,

Priestley, M.B. (1981). Spectral Analysis and Time Series. New York: Academic Press.

RAO, C.R. (1973). Linear Statistical Inference and its Applications. Second Edition. New York: Wiley.

Sacks, J., And Ylvisacker, D. (1981). Asymptotically Optimum Kernels for Density Estimation at a Point. Annals of Statistics, 9, 334-346.

Serfling, R. (1980). Approximation Theorems of Mathematical Statistics. New York: Wiley.

ShaO, J. And Tu, D. (1995). The Jackknife and Bootstrap. New York: Springer.

Shao, X. (2010a). Extended Tapered Block Bootstrap. Statistica Sinica, 20, 807-821. 
Shao, X. (2010b). The Dependent Wild Bootstrap. Journal of the American Statistical Association, 105, 489, 218-235.

Shi, X., And Shao, J. (1988). Resampling Estimation when the Observations are m-Dependent. Communications in Statistics, A, 17, 3923-3934.

Smith, R.J. (1997). Alternative Semi-Parametric Likelihood Approaches to Generalised Method of Moments Estimation. Economic Journal, 107, 503-19.

Smith, R.J. (2005). Automatic Positive Semidefinite HAC Covariance Matrix and GMM Estimation. Econometric Theory, 21, 158-170.

Smith, R.J. (2011). GEL Criteria for Moment Condition Models. Econometric Theory, $27,1192-1235$.

Sun, Y., Phillips, P.C.B. And Jin S. (2008). Optimal Bandwidth Selection in Heteroscedasticity-Autocorrelation Robust Testing. Econometrica, 76, 175-194.

Vuong, Q.H. (1989). Likelihood Ratio Tests for Model Selection and Non-Nested Hypotheses. Econometrica, 57, 307-333.

White, H. (1984). Asymptotic Theory for Econometricians. New York: Academic Press.

White, H. (1994). Estimation, Inference, and Specification Analysis. New York: Cambridge University Press 


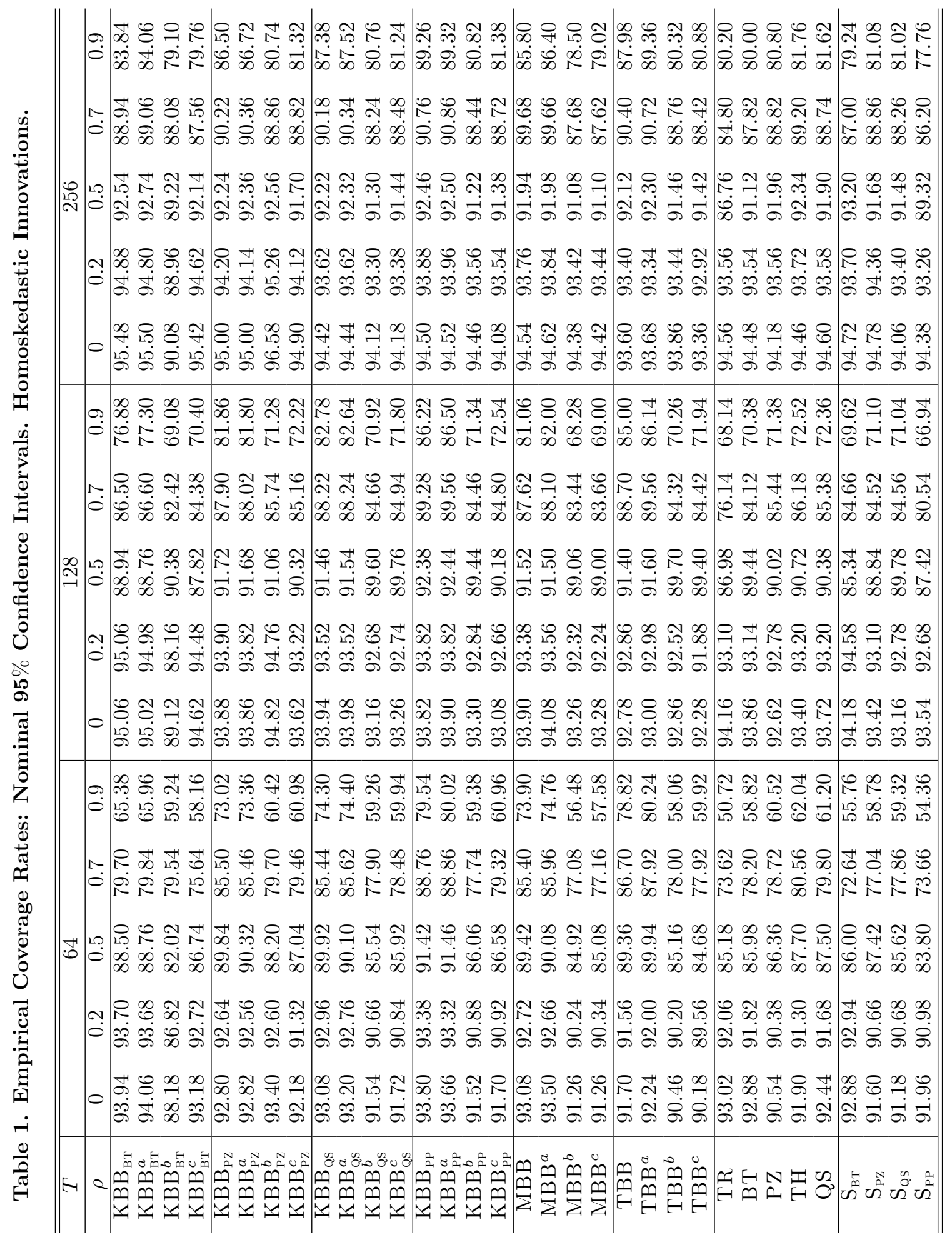




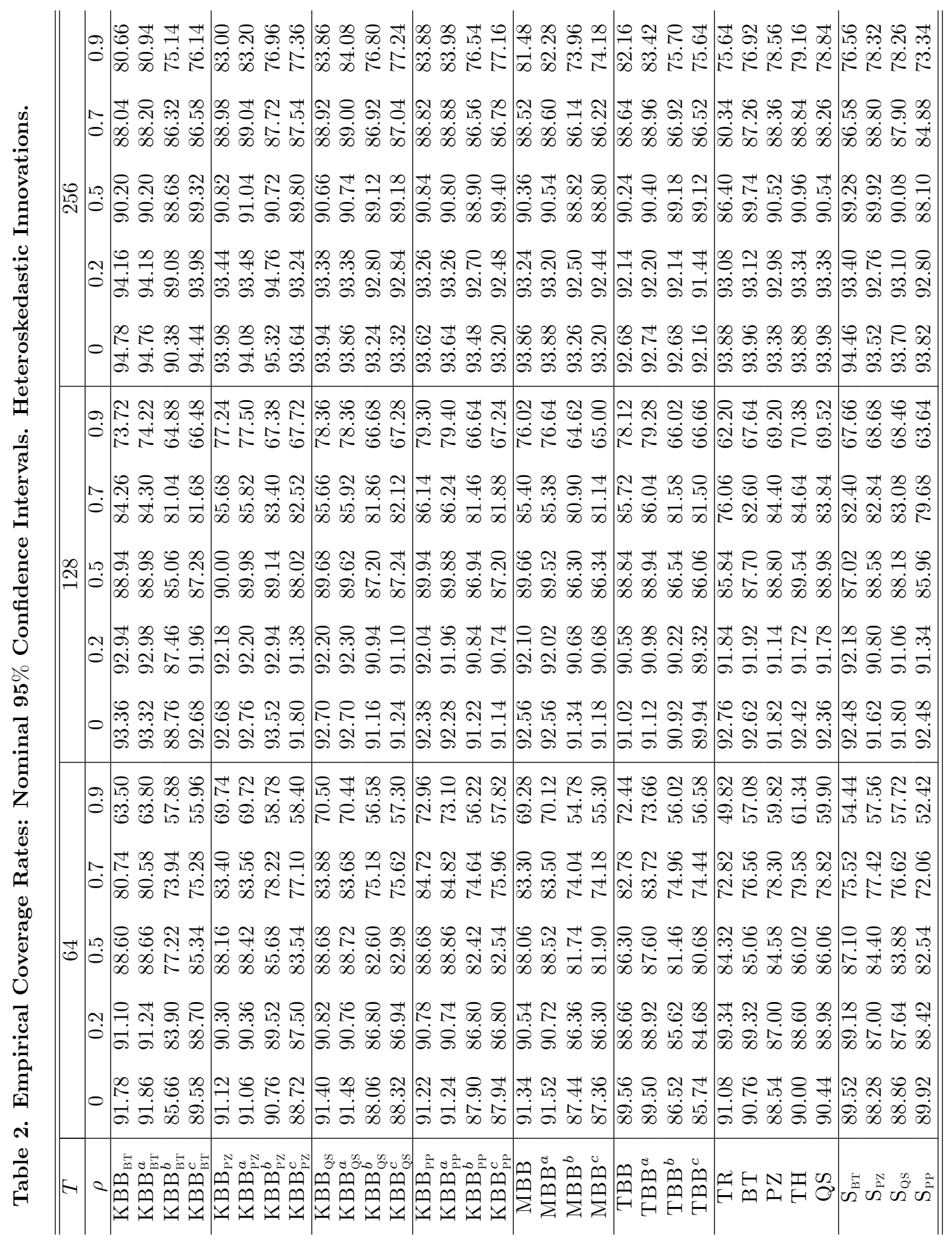




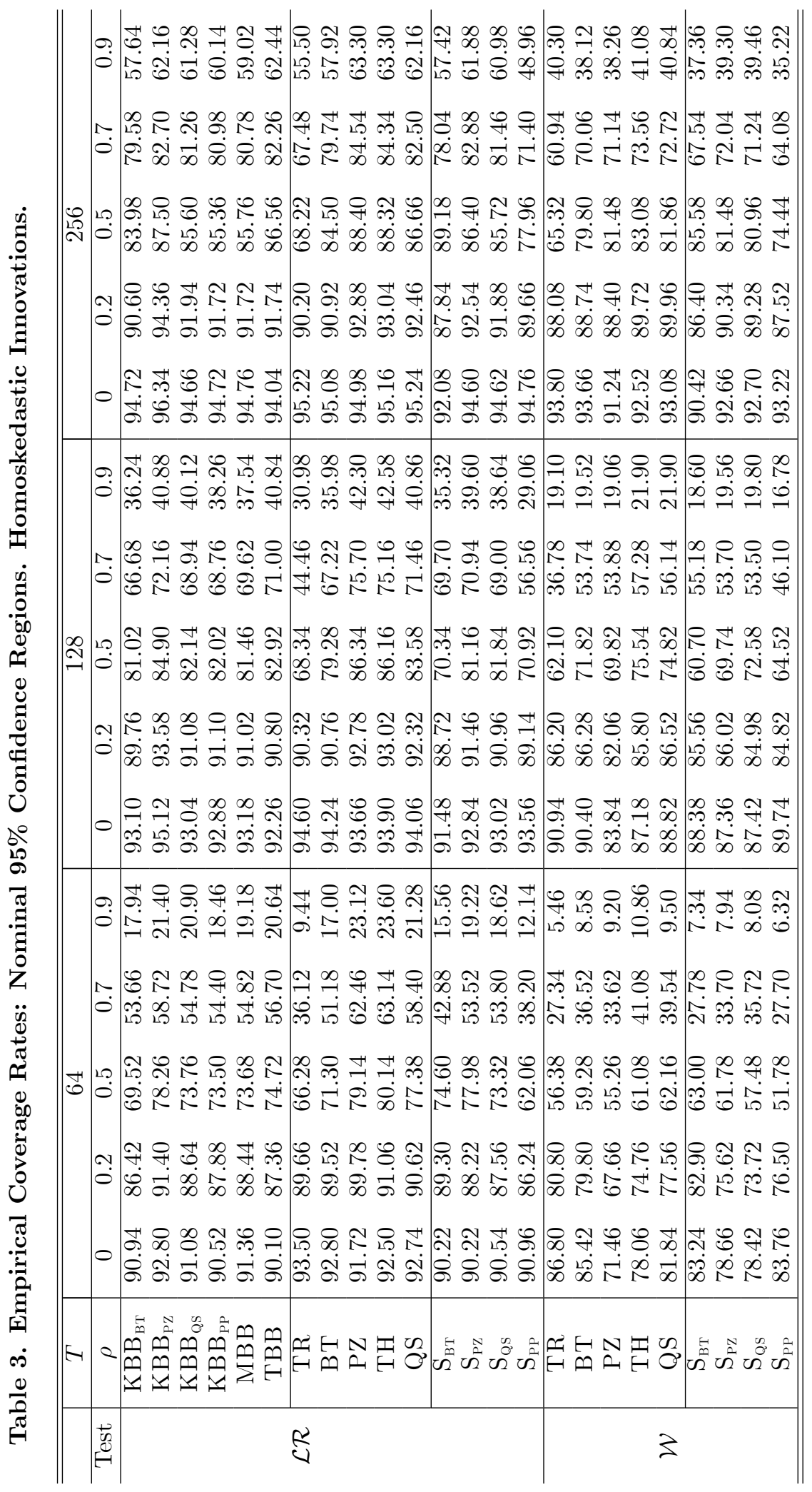




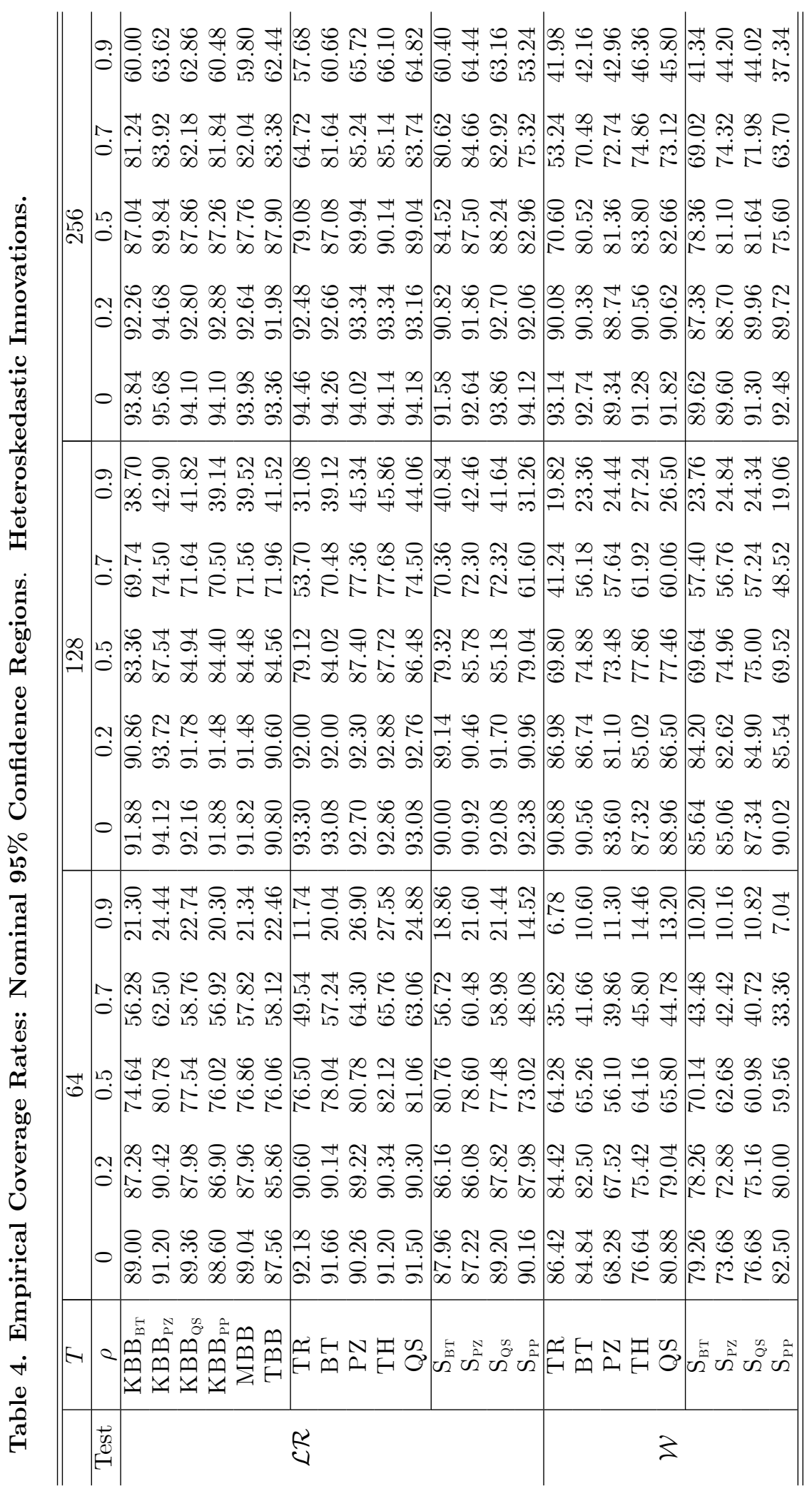




\section{University Library}

\section{- M M N E R VA A gateway to Melbourne's research publications}

Minerva Access is the Institutional Repository of The University of Melbourne

Author/s:

Parente, PMDC;Smith, RJ

Title:

QUASI-MAXIMUM LIKELIHOOD AND THE KERNEL BLOCK BOOTSTRAP FOR NONLINEAR DYNAMIC MODELS

Date:

2021

Citation:

Parente, P. M. D. C. \& Smith, R. J. (2021). QUASI-MAXIMUM LIKELIHOOD AND THE KERNEL BLOCK BOOTSTRAP FOR NONLINEAR DYNAMIC MODELS. Journal of Time Series Analysis, 42 (4), pp.377-405. https://doi.org/10.1111/jtsa. 12573.

Persistent Link:

http://hdl.handle.net/11343/276655 